\title{
Effective response theory for Floquet topological systems
}

\author{
Paolo Glorioso $\odot,{ }^{1}$ Andrey Gromov, ${ }^{2}$ and Shinsei Ryu ${ }^{1,3}$ \\ ${ }^{1}$ Kadanoff Center for Theoretical Physics, University of Chicago, Chicago, Illinois 60637, USA \\ ${ }^{2}$ Department of Physics, Brown University, Providence, Rhode Island 02912, USA \\ ${ }^{3}$ James Franck Institute, University of Chicago, Chicago, Illinois 60637, USA
}

(Received 1 November 2019; accepted 3 December 2020; published 8 February 2021)

\begin{abstract}
We present an effective field theory approach to the topological response of Floquet systems with symmetry group $G$. This is achieved by introducing a background $G$ gauge field in the Schwinger-Keldysh formalism, which is suitable for far from equilibrium systems. We carry out this program for chiral topological Floquet systems (anomalous Floquet-Anderson insulators) in two spatial dimensions and the group cohomology models of topological Floquet unitaries. These response actions serve as many-body topological invariants for topological Floquet unitaries. The effective action approach also leads us to propose topological response functions that were not considered before. For a particular family of models, we find that our response theory captures a symmetry protected version of the chiral unitary index.
\end{abstract}

DOI: 10.1103/PhysRevResearch.3.013117

\section{INTRODUCTION}

Topological phenomena in periodically driven systems (Floquet systems) have been widely discussed recently. For recent review articles, see, e.g., Refs. [1-3]. In a typical setup, we consider dynamics governed by the Hamiltonian which depends periodically on time $t: H(t+T)=H(t)$. Correspondingly, we consider the time-evolution operator

$$
U\left(t, t_{0}\right)=T \exp \left[-i \int_{t_{0}}^{t} d t^{\prime} H\left(t^{\prime}\right)\right],
$$

where $T$ represents time ordering. As a slight variation of the problem, we also consider a periodic time evolution described by a periodic unitary $U(t+T)=U(t)$, without mentioning Hamiltonians.

It has been discovered that such periodic drive can give rise to topological phenomena of at least two different kinds: (i) The periodic drive can turn a nontopological static system into a topological system, which can essentially be understood as a static topological system. (ii) The periodic drive can give rise to a topological phenomenon, which is unique to periodically driven systems and has no analog in static systems. Initial studies of topological Floquet systems were limited to the first kind of dynamical topological phenomena [4-8]. On the other hand, phenomena of the second kind have been discovered and studied more recently [9-19]. Of particular interest in this paper are topological chiral Floquet drives (anomalous Floquet-Anderson insulators) in two spatial dimensions [20-26], which are characterized by the threedimensional (3D) winding number of their single-particle Floquet unitary operators.

Published by the American Physical Society under the terms of the Creative Commons Attribution 4.0 International license. Further distribution of this work must maintain attribution to the author(s) and the published article's title, journal citation, and DOI.
The purpose of this paper is to develop an effective response field theory approach to Floquet topological systems. Our primary focus will be topological phenomena of the second kind which are intrinsic to the nonequilibrium nature of periodically driven systems.

For "static" topological phases of matter, their descriptions in terms of effective response field theories have been well developed (see, for example, Ref. [27]). A canonical example is the Chern-Simons effective field theory describing the response of quantum Hall states in $(2+1) \mathrm{D}$. One first introduces suitable background gauge fields; for the case of particle-number-conserving systems, we can introduce the background $U(1)$ gauge field $A$. We can then integrate out the dynamical "matter" fields:

$$
Z[A]=\operatorname{Tr}\left[e^{-\int_{0}^{\beta} d \tau H(A)}\right] \equiv \exp \left(-S_{\text {eff }}[A]\right),
$$

where we are working with Euclidean signature. For integer quantum Hall systems, it is known that the topological part of the effective action is purely imaginary and given by the Chern-Simons term

$$
S_{\text {eff }}[A]=\frac{i \nu}{4 \pi} \int A \wedge d A=\frac{i \nu}{4 \pi} \int d \tau d^{2} r \varepsilon^{\mu \nu \lambda} A_{\mu} \partial_{\nu} A_{\lambda},
$$

where $v$ is an integer. The modulus of the (topological part of the) partition function is independent of $A$ and can be normalized to be $1,|Z[A]| \simeq 1$.

Topological effective response field theories describe the properties of quantum many-body systems which are stable against interactions. It should be also emphasized that starting from effective response field theories it is often possible to construct explicit formulas for many-body topological invariants. (See, for example, Refs. [28,29]). In deriving the effective field theory, it is crucial that we deal with gapped (topological) phases, where matter fields represent "fast" degrees of freedom and can then be "safely" integrated over, i.e., the integration over the matter field can be controlled by the inverse gap expansion and leads to a local effective action. 
It should also be noted that in the presence of the gap, the topological term in the response field theory encodes purely the properties of the ground state. In other words, the presence or absence of the topological term in the response theory can be deduced from the adiabatic response of the ground state, without discussing gapped excited states. For example, in the case of the integer quantum Hall effect, the coefficient of the Chern-Simons term is expressed in terms of the many-body Chern number.

In this paper, we develop an effective response theory approach for periodically driven (topological) systems, paralleling effective response theories for static topological phases with symmetry. Specifically, we will work with the Schwinger-Keldysh generating functional of Floquet unitaries,

$$
Z\left[A_{1}, A_{2}\right]=\operatorname{Tr}\left[U\left(A_{1}\right) \rho_{0} U^{\dagger}\left(A_{2}\right)\right],
$$

where we have introduced external (nondynamical) $U(1)$ gauge fields $A_{1}$ and $A_{2}$, which couple to the evolution operator $U(t)$ and to its conjugate $U^{\dagger}(t)$, respectively. ${ }^{1}$ The initial state $\rho_{0}$, will be taken to be a Gibbs ensemble at infinite temperature, $\rho_{0} \sim e^{\alpha Q}$, where $\alpha$ is a chemical potential and $Q$ is the $U(1)$ charge operator. We will demonstrate that the Schwinger-Keldysh generating functional $W\left[A_{1}, A_{2}\right]=$ $-i \log Z\left[A_{1}, A_{2}\right]$ for many-body localized Floquet systems is a local functional of $A_{1}$ and $A_{2}$, and, furthermore, encodes the topology of the system. In fact, we will see that this framework can be applied to many-body localized systems whose explicit time dependence is not necessarily periodic, as the topological origin of our response functional-i.e., its independence on smooth deformations of the system-is completely unrelated to time periodicity of the microscopic system. Periodicity, combined with other properties, will only be used to show quantization of topological response.

The paper is organized as follows. In Sec. II, we explain how to apply the Schwinger-Keldysh approach to periodically driven topological systems. In Sec. III, we consider chiral Floquet drives (anomalous Floquet Anderson insulators) in two spatial dimensions, for which we explicitly compute the Schwinger-Keldysh effective action, and identify the topological term. We also show that, for a particular family of models, this topological term captures a symmetry protected version of the chiral unitary index discussed in $[24,44]$. In Sec. IV, we describe two generalizations of topological effective response that were not considered before. Further material and technical details are discussed in the Appendix. In Appendix A, we study yet another class of topological Floquet drives, those which are constructed by using the group cohomology. There, we find that the topological terms of the Schwinger-Keldysh functional are members of (labeled by) $H^{d}(G, U(1))$, where $G$ is the symmetry group and $d$ is the spatial dimension. In Appendix B, we discuss an approach based on the so-called channel-state map, which provides a perspective complementary to the Schwinger-Keldysh approach.

\footnotetext{
${ }^{1}$ One can also promote static background gauge fields to dynamical ones, by integrating over the gauge field. The effect of such dynamical gauging was discussed in Ref. [18]. In this work, we will confine ourselves to background gauge fields.
}

\section{SCHWINGER-KELDYSH RESPONSE}

\section{A. Generalities}

In this section, we introduce the basic framework that will be used as a systematic approach to topological Floquet phases. While our interest lies in Floquet systems, we shall start with general discussions that can be applied to any timedependent Hamiltonian $H(t)$. A modern introduction to the Schwinger-Keldysh formalism can be found in Refs. [30,31].

We will assume that $H(t)$ possesses a $U(1)$ symmetry, and we couple it to an external gauge field $A_{\mu}(t, \vec{r})$, so that the evolution operator is given by

$$
U\left(t_{1}, t_{0} ; A\right)=T \exp \left[-i \int_{t_{0}}^{t_{1}} d t^{\prime} H\left(t^{\prime} ; A\right)\right],
$$

where $H(t ; A)$ is the Hamiltonian coupled to $A_{\mu}(t, \vec{r})$. The current conjugate to $A_{\mu}$ will be denoted as $J^{\mu}$.

We introduce the Schwinger-Keldysh generating functionals $Z\left[A_{1}, A_{2}\right]$ and $W\left[A_{1}, A_{2}\right]$ by $[30,31]$

$$
Z\left[A_{1}, A_{2}\right]=e^{i W\left[A_{1}, A_{2}\right]}=\operatorname{Tr}\left[U\left(t_{1}, t_{0} ; A_{1}\right) \rho_{0} U^{\dagger}\left(t_{1}, t_{0} ; A_{2}\right)\right],
$$

where $\rho_{0}$ is the initial state of the system at $t=t_{0}$. The operator inside the trace can be thought of as the time evolution of the density matrix $\rho_{0}, \rho\left(t_{1}\right)=U\left(t_{1}, t_{0} ; A_{1}\right) \rho_{0} U^{\dagger}\left(t_{1}, t_{0} ; A_{2}\right)$, where each factor of the evolution is coupled to a different gauge field $A_{1 \mu}$ and $A_{2 \mu}$.

In typical applications, we adiabatically switch on perturbations causing nonequilibrium dynamics. It is then convenient to start the time evolution from $t_{0}=-\infty$ with the initial state $\rho_{0}$ in the remote past which is chosen as an equilibrium state. We also send $t_{1} \rightarrow+\infty$ when discussing correlation functions with operators located at arbitrary late times. Then, the Schwinger-Keldysh contour runs from $-\infty$ to $+\infty$ and back. One striking feature is that this approach does not require knowing the final state

The Schwinger-Keldysh trace with background (6) provides a compact and efficient way to encode various nonequilibrium correlation functions. Indeed, differentiating $Z\left[A_{1}, A_{2}\right] n$ times with respect to $A_{1 \mu}$ and $m$ times with respect to $A_{2 \mu}$ leads to a correlation function of $n$ time-ordered and $m$ antitime-ordered currents $J^{\mu}$

$$
\begin{aligned}
\operatorname{Tr} & {\left[\rho_{0} T\left(J^{\mu}\left(x_{1}\right) \cdots\right) \tilde{T}\left(J^{\alpha}\left(x_{n+1}\right) \cdots\right)\right] } \\
& =\left.\frac{1}{i^{n}(-i)^{m}} \frac{\delta^{n+m} e^{i W\left[A_{1}, A_{2}\right]}}{\delta A_{1 \mu}\left(x_{1}\right) \cdots \delta A_{2 \alpha}\left(x_{n+1}\right) \cdots}\right|_{A_{1,2}=0},
\end{aligned}
$$

where $x=(t, \vec{r})$ and $\tilde{T}$ represents antitime ordering.

The generating functional $W\left[A_{1}, A_{2}\right]$ should satisfy certain basic properties due to unitarity of the evolution:

$$
\begin{array}{r}
W\left[A_{1}, A_{2}\right]=-W^{*}\left[A_{2}, A_{1}\right], \quad W[A, A]=0, \\
\operatorname{Im} W\left[A_{1}, A_{2}\right] \geqslant 0,
\end{array}
$$

where the first two can be seen straightforwardly from the definition (6), while the last condition follows from the fact that the absolute value of the trace of the operator $U\left(\infty,-\infty ; A_{1}\right) \rho_{0} U^{\dagger}\left(\infty,-\infty ; A_{2}\right)$ is bounded by unity $[31,32]$. 


\section{B. Application to (topological) Floquet systems}

We will now apply the Schwinger-Keldysh formalism to study topological properties of Floquet systems.

\section{Choice of the initial state}

For static systems, one typically chooses the initial state $\rho_{0}$ to be the ground state or the thermal state. In the case of our interest, we observe that the time dependence of the Hamiltonian is not slow compared to the energy gap of the instantaneous Hamiltonian $H(t)$, and thus there is no notion of ground state or of thermal equilibrium. The most natural choice in this context, in the absence of any symmetry, is to choose $\rho_{0}$ to be the infinite temperature state,

$$
\rho_{0}=I / \mathcal{N}
$$

where $I$ is the identity operator and the normalization factor $\mathcal{N}$ is the dimension of the Hilbert space. In Appendix B, we will see that with this choice of initial state, the SchwingerKeldysh trace can be viewed as an inner product of unitaries when unitaries are mapped to states by using the channel-state map (the so-called Choi-Jamiołkowski isomorphism).

In the presence of a symmetry, the most natural choice of $\rho_{0}$ is the Gibbs ensemble formed by the conserved charges of the system,

$$
\rho_{0}=\frac{e^{\alpha Q}}{\operatorname{Tr} e^{\alpha Q}},
$$

where $Q$ is the charge operator (number operator) associated to the $U(1)$ symmetry, and the parameter $\alpha$ plays the role of chemical potential. Instead of introducing $\alpha$ in the initial state $\rho_{0}, \alpha$ can also be introduced as the difference between the (uniform and time-independent) temporal component of $A_{1}$ and $A_{2}$ in $U\left(t_{1}, t_{0} ; A_{1}\right)$ and $U\left(t_{1}, t_{0} ; A_{2}\right)$.

This choice of initial state allows us to put our focus on properties of evolution operators themselves rather than the time evolution of individual states. (See, for example, Ref. [33] which also uses the infinite temperature state). We also recall that under Floquet time evolution, states may indefinitely be heated up by the drive, which may wash out any topological phenomena. Various mechanisms in the literature are used to prevent this (e.g., many-body localization [34-36] or prethermalization [37-42]). It is also worth recalling that eigenstates of Floquet unitaries are all expected to behave similarly, e.g., no mobility gap separating ergodic and many-body localized states.

\section{Choice of the Schwinger-Keldysh contour}

We now describe our choice of the Schwinger-Keldysh contour. First, we note that there are characteristic values of times, integer multiples of the period of the Floquet drive $T$. In our discussion, we will mostly evaluate the SchwingerKeldysh generating functional for $t_{1}-t_{0}=$ (integer) $\times T$. At these values the generating functional will exhibit additional important properties in relation to topology when dealing with special models-see Sec. III.

Second, for generic systems, it will be important to take the integers $m, n$ to be large. A convenient object to study response to $A_{\mu}$ is then

$$
\begin{aligned}
Z\left[A_{1}, A_{2}\right] & =e^{i W\left[A_{1}, A_{2}\right]} \\
& =\lim _{\kappa \rightarrow \infty} \operatorname{Tr}\left[U\left(\kappa T,-\kappa T ; A_{1}\right) \rho_{0} U^{\dagger}\left(\kappa T,-\kappa T ; A_{2}\right)\right],
\end{aligned}
$$

where we chose $t_{1}=-t_{0}=\kappa T$ with $\kappa$ a half-integer; for simplicity, we have chosen the Schwinger-Keldysh contour to be symmetric around $t=0$. As we will see, the infinite-time limit guarantees that, for generic models, when the system is in the localized regime, only topological contributions will survive, as in the infinite-time limit nontopological effects are averaged out, making them transparent to the response captured by the generating functional $W\left[A_{1}, A_{2}\right]$.

Having fixed the definition of the time contour, we now discuss the structure of the gauge transformations. These have the form

$$
A_{1 \mu} \rightarrow A_{1 \mu}+\partial_{\mu} \lambda_{1}, \quad A_{2 \mu} \rightarrow A_{2 \mu}+\partial_{\mu} \lambda_{2},
$$

where $\lambda_{1}(t, \vec{r})$ and $\lambda_{2}(t, \vec{r})$ are independent functions, except at the end points $t_{0}$ and $t_{1}$, where they must be related as

$$
\begin{aligned}
& \lambda_{1}\left(t_{0}, \vec{r}\right)=\lambda_{2}\left(t_{0}, \vec{r}\right)+2 \pi n_{0}, \\
& \lambda_{1}\left(t_{1}, \vec{r}\right)=\lambda_{2}\left(t_{1}, \vec{r}\right)+2 \pi n_{1},
\end{aligned}
$$

where $n_{0}$ and $n_{1}$ are integers. Small gauge transformations will satisfy $\lambda_{1}, \lambda_{2} \rightarrow 0$ at $t=t_{0}, t_{1}{ }^{2}$

The gauge invariance of the effective action will be further discussed in Sec. III A.

\section{Slowly varying background}

We will restrict to background sources which are slowly varying in space and time. In our discussion, we will be concerned with systems which are in the localized regime. As far as the system localizes, we expect the generating functional $W$ to be a local functional in $A_{1}$ and $A_{2}$, which is a crucial feature of our formulation as it will allow us to write down $W$ in a derivative expansion in $A_{1}$ and $A_{2}$, as far as the latter are sufficiently slowly varying, enabling us to identify particular couplings in $W$ which contribute to topological response.

Below we will be interested in the structure of the Schwinger-Keldysh generating functional $W$, which depends on background $U(1)$ gauge fields $A_{1 \mu}, A_{2 \mu}$ and on the constant chemical potential $\alpha$. For the rest of the paper, we will further take $A_{0}=0$ and $A_{i}=A_{i}(\vec{r})$, for both copies of the background. We will thus restrict to "static" response. Operationally, these configurations are the most general for which

\footnotetext{
${ }^{2} \mathrm{We}$ assume that, in the operator formalism, gauge transformations are implemented by unitary transformations of the form $V(t)=$ $e^{i \sum_{r} \lambda(t, r) n_{r}}$, where $n_{r}$ is the charge density operator. The evolution operators in the Schwinger-Keldysh generating functional transform as

$$
\begin{aligned}
& U\left(t_{1}, t_{0} ; A_{1}\right) \rightarrow V_{1}\left(t_{1}\right) U\left(t_{1}, t_{0} ; A_{1}\right) V_{1}^{\dagger}\left(t_{0}\right), \\
& \text { and } U\left(t_{1}, t_{0} ; A_{2}\right) \rightarrow V_{2}\left(t_{1}\right) U\left(t_{1}, t_{0} ; A_{2}\right) V_{2}^{\dagger}\left(t_{0}\right),
\end{aligned}
$$

which implies Eq. (13).
} 
we can analytically compute topological response from the microscopic models that we are interested in, and take the continuum limit. We will see that this choice is sufficient to capture the topological character of periodically driven systems. ${ }^{3}$

It is convenient to introduce a new basis for the background,

$$
A_{r i}=\frac{1}{2}\left(A_{1 i}+A_{2 i}\right), \quad A_{a i}=A_{1 i}-A_{2 i},
$$

where this change of basis is sometimes referred to as the Keldysh rotation [30]. This basis is convenient as the constraints (8) can be easily implemented. We will write $W$ as an expansion in number of derivatives acting on $A_{r i}, A_{a i}$, and a power expansion in $A_{a i}$, which will make it easy to enumerate the list of terms compatible with (8). Note that the second condition in (8) requires each term in $W$ to contain at least one power of $A_{a i}$. To zeroth order in derivatives, there is no gauge-invariant term that we can write down. To first order in derivatives, the most general generating functional is

$$
W=i \frac{\Theta(\alpha)}{2 \pi T} \int d t d^{2} r B_{a}, \quad B_{a}=\varepsilon^{i j} \partial_{i} A_{a j},
$$

where $\Theta(\alpha)$ is an arbitrary function of $\alpha$. One immediately sees that (17) satisfies conditions (8). Additional terms will be at least second order in derivatives, such as $\left(\varepsilon^{i j} \partial_{i} A_{a j}\right)^{2}$ or $\left(\varepsilon^{i j} \partial_{i} A_{r j}\right)\left(\varepsilon^{k l} \partial_{k} A_{a l}\right)$. Since we are interested in topological responses, we will focus on (17), as it is the only term with a coupling constant that is dimensionless in length units. In the next sections we will focus on a family of systems which displays precisely this type of response, and we will see how their topological properties are encoded in the function $\Theta(\alpha)$. We will look at Floquet systems defined on closed as well as open spatial manifolds. In the first case, we will consider backgrounds with nontrivial flux in order for (17) to contribute, while in the second case (17) can be written as a boundary term.

\section{TOPOLOGICAL CHIRAL FLOQUET DRIVE}

In this section we shall study in detail the SchwingerKeldysh Floquet response of a particular model. Consider a two-dimensional square lattice with periodic boundary conditions of size $L_{x} \times L_{y}$, where $L_{x}, L_{y}$ are even integers. The total number of sites is $L_{x} L_{y}=N$. We denote site coordinates with $r=(x, y) \in \mathbb{Z} \times \mathbb{Z}$, and split sites into sublattice $A$, with coordinates $x+y \in 2 \mathbb{Z}$, and sublattice $B$ with coordinates $x+y \in 2 \mathbb{Z}+1$. The model is given by a Floquet Hamiltonian $H(t)$ of period $T$ obtained as follows. Divide the period $T$ in five intervals of equal duration $T / 5$, where each of the first four intervals has Hamiltonian $H_{n}$, with $n=1,2,3$, 4, where

$$
H_{n}=\sum_{r \in A} H_{n, r}, \quad H_{n, r}=-J\left(e^{i A_{r, r+b_{n}}} c_{r}^{\dagger} c_{r+b_{n}}+\text { H.c. }\right) \text {, }
$$

with $J=\frac{2.5 \pi}{T}$, and where

$$
b_{1}=-b_{3}=(1,0), \quad b_{2}=-b_{4}=(0,1),
$$

\footnotetext{
${ }^{3}$ We remark that, from the point of view of our effective response, there is no technical limitation in considering terms which depend on $A_{10}, A_{20}$, and which have (slow) time dependence. We leave this to future work.
}
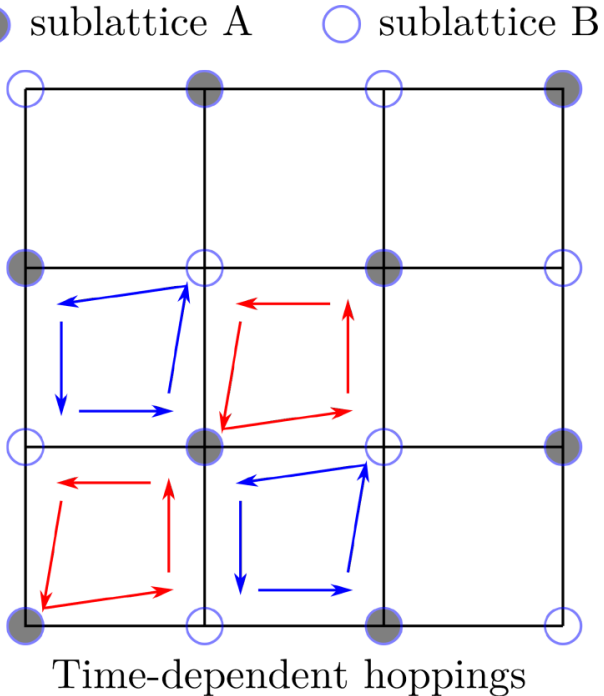

FIG. 1. The topological chiral Floquet drive (18) on the square lattice. Red and blue arrows represent chiral trajectories of particles starting from A and B sublattice, respectively.

while during the fifth interval the Hamiltonian is zero. The fifth interval will be of practical use later, when we shall introduce disorder. Note that the $H_{n, r}$ and $H_{n, r^{\prime}}$ commute with each other, so the evolution can be factorized on each site $r \in A$. The resulting evolution is to move a particle around a plaquette, and bring it back to its original position after one period, as illustrated in Fig. 1. This model was originally introduced in Ref. [20] and has been extensively studied, e.g., in Refs. [21,22]. In addition, we added a minimal coupling to a background $U(1)$ gauge field, where

$$
A_{r, r+b}=\int_{r}^{r+b} d r A(r)
$$

is the gauge link from site $r$ to site $r+b$. Note that, as mentioned in Sec. II, we are restricting to background gauge fields with $A_{0}=0$ and $A_{i}=A_{i}(r)$. In principle, one can take slowly time-dependent background sources $A_{i}(t, \vec{r})$ and perturbatively solve the model by doing derivative expansion in time. However, we expect such configurations to contribute only through higher derivatives to the generating functional $W$. Static configurations will be sufficient to evaluate $\Theta(\alpha)$ introduced in (17), thus capturing the topological character of these periodically driven systems.

The Floquet unitary is given by

$$
U(T / 2,-T / 2) \equiv U_{F}=U_{4} U_{3} U_{2} U_{1}, \quad U_{n}=\prod_{r} e^{-i T / 5 H_{n, r}}
$$

with $e^{-i T / 5 H_{n, r}}=1-\left(n_{r}-n_{r+b_{n}}\right)^{2}+i\left(n_{r}-n_{r+b_{n}}\right)^{2}\left(e^{i A_{r, n}} c_{r}^{\dagger}\right.$ $c_{r+b_{n}}+$ H.c.). In passing, we notice that this model has a unitary on-site particle-hole symmetry, given by

$$
c_{r} \rightarrow(-1)^{r} c_{r}^{\dagger}, \quad c_{r}^{\dagger} \rightarrow(-1)^{r} c_{r}, \quad A_{i}(r) \rightarrow-A_{i}(r),
$$

where $(-1)^{r}=+1$ or -1 if $r$ belongs to sublattice $A$ or $B$, respectively.

This Floquet drive is special or ideal in the sense that, in the absence of the background gauge field, $U_{F}=I$, i.e., the Floquet Hamiltonian is identically zero, and hence there is 
no heating. For more generic models, this is not the case and $U_{F}=\exp \left(-i T H_{F}\right)$, where $H_{F}$ is a Floquet Hamiltonian. To avoid heating, we need to demand $H_{F}$ is, e.g., many-body localizing.

\section{A. Response functional on the torus}

We shall now compute the Schwinger-Keldysh generating functional $W\left[A_{1}, A_{2}\right]$ introduced in (6) for the chiral Floquet drive, subject to the periodic boundary conditions described in the beginning of this section. Since the Hamiltonian is quadratic ( $U$ is Gaussian), $W$ reduces to a quantity built from the single-particle counterpart of the Floquet unitary; $U\left(t_{1}, t_{0}\right)$ transforms the fermion creation or annihilation operators as $U\left(t_{1}, t_{0}\right) c_{i} U^{\dagger}\left(t_{1}, t_{0}\right)=\mathcal{U}_{i j}\left(t_{1}, t_{0}\right) c_{j}$ and $U\left(t_{1}, t_{0}\right) c_{i}^{\dagger} U^{\dagger}\left(t_{1}, t_{0}\right)=$ $\mathcal{U}_{i j}^{*}\left(t_{1}, t_{0}\right) c_{j}^{\dagger}=c_{j}^{\dagger} U_{j i}^{\dagger}\left(t_{1}, t_{0}\right)$, where the $N \times N$ unitary matrix $\mathcal{U}_{i j}\left(t_{1}, t_{0}\right)$ is the single-particle evolution operator acting on the single-particle Hilbert space. By noting the formula

$$
\operatorname{Tr}\left(e^{\sum_{i, j} c_{i}^{\dagger} \mathcal{A}_{i j} c_{j}}\right)=\operatorname{det}\left(I+e^{\mathcal{A}}\right),
$$

we then find

$$
e^{i W}=\frac{1}{\operatorname{Tr}\left(e^{\alpha Q}\right)} \operatorname{det}\left[e^{-\frac{\alpha}{2}} I+e^{\frac{\alpha}{2}} \mathcal{U}\left(A_{1}\right) \mathcal{U}^{\dagger}\left(A_{2}\right)\right]
$$

where we used (10) as initial density matrix, and $\mathcal{U}(A) \equiv$ $\mathcal{U}(\kappa T,-\kappa T ; A)$. (Here and henceforth, "tr" and "det" denote the trace and determinant in the $N$-dimensional single-particle Hilbert space, respectively, as opposed to "Tr" which is the trace taken over the $2^{N}$-dimensional many-body Hilbert space). We used a "particle-hole symmetrized" definition of number operator, i.e.,

$$
Q=\sum_{r}\left(n_{r}-1 / 2\right), \quad n_{r}=c_{r}^{\dagger} c_{r}
$$

where the eigenvalues of $Q$ run from $-\frac{N}{2}$ to $\frac{N}{2}$, and $\operatorname{Tr}\left(e^{\alpha Q}\right)=$ $\prod_{r}\left(e^{-\frac{\alpha}{2}}+e^{\frac{\alpha}{2}}\right)=\left(2 \cosh \frac{\alpha}{2}\right)^{N}$. The chemical potential $\alpha$ can be used to project the "unnormalized" generating functional to a given sector with fixed particle number:

$$
\operatorname{Tr}\left(e^{\alpha Q}\right) e^{i W}=\sum_{q} e^{\alpha q} Z_{q+N / 2}\left[A_{1}, A_{2}\right],
$$

where the subscript $q+N / 2$ is the nonsymmetrized particle number running from 0 to $N$. For the case of Gaussian Floquet unitaries, expanding the determinant in (22) we obtain, for example,

$$
\begin{aligned}
& Z_{1}\left[A_{1}, A_{2}\right]=\operatorname{tr}\left[\mathcal{U}\left(A_{1}\right) \mathcal{U}^{\dagger}\left(A_{2}\right)\right], \\
& Z_{N}\left[A_{1}, A_{2}\right]=\operatorname{det}\left[\mathcal{U}\left(A_{1}\right) \mathcal{U}^{\dagger}\left(A_{2}\right)\right] .
\end{aligned}
$$

It is easy to check that $\mathcal{U}_{F}(A)$ is diagonal with its diagonal elements given by $e^{i B_{r}}$, where $B_{r}$ is a flux picked up by a particle which is located initially at $r: \mathcal{U}_{F}(A)=\sum_{r} e^{i B_{r}}|r\rangle\langle r|$. Then,

$$
\begin{aligned}
e^{i W} & =\frac{1}{\left(2 \cosh \frac{\alpha}{2}\right)^{N}} \sum_{\left\{n_{r}=0,1\right\}} e^{\left(n_{r}-\frac{1}{2}\right) \alpha} e^{+i \int \frac{d t}{T} \sum_{r}\left(B_{1 r}-B_{2 r}\right) n_{r}} \\
& =\frac{1}{\left(2 \cosh \frac{\alpha}{2}\right)^{N}} \prod_{r}\left[e^{-\frac{\alpha}{2}}+e^{\frac{\alpha}{2}} e^{i \int \frac{d t}{T}\left(B_{1 r}-B_{2 r}\right)}\right] .
\end{aligned}
$$

Note that the time integral in the exponents $\int d t$ should be thought of as $\int_{-\kappa T}^{\kappa T} d t$, with $\kappa$ a sufficiently large integer. We can check that (26) is consistent with particle-hole symmetry,

$$
\frac{Z\left[-A_{1},-A_{2},-\alpha\right]}{Z\left[A_{1}, A_{2}, \alpha\right]}=e^{i \int \frac{d t}{T} \sum_{r}\left[B_{1 r}-B_{2 r}\right]}=1,
$$

where we noted the quantization of the total flux $\sum_{r} B_{s r}=$ $2 \pi \times$ (integer) $(s=1,2)$ on a close manifold since $A_{r, r^{\prime}}$ is an angular variable, $A_{r, r^{\prime}} \equiv A_{r, r^{\prime}}+2 \pi$.

Equation (26) is the exact microscopic result and can be used to study systems with arbitrary configurations of the background gauge fields-see around Eqs. (30) and (36), for example. We now specialize to background configurations which are slowly varying compared to the lattice constant. In this limit, $\partial A_{i} / \partial r_{j} \ll A_{i}$, i.e., one expands $e^{i B_{r}}=1+i B_{r}+$ $\cdots$, and resumming, the only finite contribution to the generating functional will be ${ }^{4}$

$$
\exp i W\left[A_{1}, A_{2}\right]=\exp i \frac{\Theta(\alpha)}{2 \pi} \int \frac{d t}{T} \int d^{2} r\left[B_{1}(r)-B_{2}(r)\right],
$$

with

$$
\Theta(\alpha)=\theta+f(\alpha), \quad \theta=\Theta(0)=\pi, \quad f(\alpha)=\pi \tanh \frac{\alpha}{2} .
$$

Note that the generating functional is now a pure phase and topological in the sense that it does not require a (spatial) metric for its definition. In Sec. III D we will show that $\Theta(\alpha)$ is independent of continuous deformations of the microscopic system, and in Sec. III E we will prove that $\theta$ is quantized.

The effective action (28) is a Schwinger-Keldysh analog of the theta term, $\exp \left[i \frac{\theta}{2 \pi} \int_{M_{2}} d A\right]$, which appears, e.g., as an effective response functional of $(1+1)$-dimensional static topological insulators (e.g., the SSH model), where $M_{2}$ is the $(1+1)$-dimensional space time [27]. For the static case, $\theta$ is a periodic variable, $\theta \equiv \theta+2 \pi$, because of the Dirac quantization condition: For any $(1+1)$-dimensional closed Euclidean space time $M_{2}, \int_{M_{2}} d A=2 \pi \times$ integer, which is a consequence of the large $U(1)$ gauge invariance.

For the Schwinger-Keldysh functional (28), the situation seems more complicated in the sense that the combination $A_{a i}=A_{1 i}-A_{i 2}$ entering in (28) is "neutral" under spatial large gauge transformations, i.e., transformations which allow a nontrivial flux of $A_{a i}$ across the torus. Indeed, due to (13), $\lambda_{1}\left(t_{0}, r\right)$ and $\lambda_{2}\left(t_{0}, r\right)$ must be topologically equivalent as spatial functions, as well as $\lambda_{1}\left(t_{1}, r\right)$ and $\lambda_{2}\left(t_{1}, r\right)$, and hence there is no large gauge transformation to quantize $\int d A_{a}=\int\left(d A_{1}-d A_{2}\right)$ (where we consider the integral only over the space, as $\int d t / T$ is simply an integer). Nevertheless, we can still argue that $\Theta(\alpha)$ in (28) has a periodicity $\Theta(\alpha) \equiv \Theta(\alpha)+2 \pi$. First we note that if the dependence on $A_{1}$ and $A_{2}$ of the generating functional $Z\left[A_{1}, A_{2}\right]$ enters through the total fluxes $\sum_{r} B_{1 r}$ and $\sum_{r} B_{2 r}$, they have to be separately quantized since $A_{1}$ and $A_{2}$ are angular variables, $A_{s r} \equiv A_{s r}+2 \pi$. Second, periodicity of $\Theta(\alpha)$ can also be

\footnotetext{
${ }^{4}$ Note that the continuum limit should be taken before the infinitetime limit, i.e., in taking $B_{r} \rightarrow 0$, the integral $\int d t$ should be performed over a finite-time interval.
} 
proven from the following argument. When we switch off one of the gauge fields, $A_{2}$, say, the Floquet unitary of the model reduces to identity $U\left(\kappa T,-\kappa T ; A_{2}=0\right)=I$, i.e., the second Schwinger-Keldysh copy simply disappears. Hence the Schwinger-Keldysh trace (6) reduces to $e^{i W\left[A_{1}, A_{2}=0\right]}=$ $\operatorname{Tr}\left[U\left(A_{1}\right) \rho_{0}\right]$ which is now invariant under the "accidental" large gauge transformation $A_{1 i} \rightarrow A_{1 i}+\partial_{i} \lambda$, while $A_{2 i}$ remains zero. ${ }^{5}$ On the other hand, the effective action (28) reduces to $\exp \left[-i \frac{\Theta(\alpha)}{2 \pi} \int \frac{d t}{T} \int d^{2} r B_{1}(r)\right]$ in which $\Theta(\alpha)$ should be now periodic because of the (large) gauge invariance under $A_{1 i} \rightarrow A_{1 i}+\partial_{i} \lambda$.

In the latter argument above, we relied on a special feature of the model, $U(\kappa T, \kappa T ; A=0)=I$, which is not true in general: For more general cases $U(\kappa T,-\kappa T ; A=0)$ is not the identity given by the exponentiated Floquet Hamiltonian, $U(\kappa T,-\kappa T ; A=0)=\exp \left(-i 2 \kappa T H_{F}\right)$. Nevertheless, the periodicity of the Theta angle $\Theta(\alpha)$ in the Schwinger-Keldysh effective action will persist at least for a wide class of models, thanks to the fact that the value of $\Theta(\alpha)$ is independent of continuous deformations of the system, as will be shown in Sec. III D. Indeed, Floquet unitaries $U(t)$ can be smoothly deformed into the form $U^{\prime}(t)=\tilde{U}(t) \exp \left(-i t H_{F}\right)$, where $\tilde{U}(t)$ is periodic (the so-called micromotion part), with $\tilde{U}(t=T)=$ $I$, and $\exp \left(-i t H_{F}\right)$ captures the nonperiodic part. If one can then smoothly deform $U(t)$ into $\tilde{U}(t)$ (see, e.g., Ref. [19]), which one can do with the nonperiodic evolutions of the models discussed in later sections, ${ }^{6}$ then the identification $\Theta(\alpha)=\Theta(\alpha)+2 \pi$ continues to hold. This implies that, on the torus, $\theta$ is a $\mathbb{Z}_{2}$ invariant. We will see in Sec. IIIC that, taking the Schwinger-Keldysh trace on a strip, $\theta$ is unambiguous and therefore defines a $\mathbb{Z}$ invariant.

In Sec. IIIE we will prove that $\theta$ is quantized for generic Hamiltonians. In the case of the simple model (18) and (19), quantization of $\theta$ can also be easily implied using the accidental particle-hole symmetry discussed around Eq. (21), $\left(A_{1 i}, A_{2 i}, \alpha\right) \rightarrow\left(-A_{1 i},-A_{2 i},-\alpha\right)$, i.e.,

$$
\begin{aligned}
& \exp i W\left[A_{1}, A_{2}\right] \\
& \quad \rightarrow \exp -i \frac{\Theta(-\alpha)}{2 \pi} \int \frac{d t}{T} \int d^{2} r\left[B_{1}(r)-B_{2}(r)\right],
\end{aligned}
$$

which means that, when $\alpha=0, \theta$ is quantized as $\theta=\pi \times$ integer. As in the case of static topological insulators, the quantized theta term can be thought of as a topological invariant differentiating topologically distinct (many-body localized) Floquet unitaries (regardless of the microscopic details of the system, and even for strongly coupled many-body systems, as far as the thermodynamic limit is well defined). For generic values $\alpha$, one can see that particle-hole symmetry implies $f(\alpha)=-f(-\alpha)$. In the next section, we will show that $\Theta(\alpha)$ is independent of continuous deformations of the Hamiltonian and that $f(\alpha)$ contains additional topological information of the system.

\footnotetext{
${ }^{5}$ This enhanced symmetry can also be seen without setting $A_{2}=0$ by simply noting that $U\left(A_{2}\right)$, for this particular model, commutes with gauge transformations.

${ }^{6}$ In fact, some literature just entirely focuses on periodic Floquet unitaries $U(0)=U(T)=I$, see Ref. [19].
}

We close this subsection with a few remarks. First, while we have been focusing on smooth configurations of the background gauge fields, it is also interesting to consider nonsmooth configurations, e.g., a pair of localized magnetic fluxes $\phi$ and $-\phi$ inserted through two plaquettes. The corresponding background gauge field can be introduced by considering a "string" on the dual lattice connecting these two plaquettes and assigning $e^{i A_{r r^{\prime}}}=e^{ \pm i \phi}$ for those links intersecting the string. It is straightforward to see

$$
Z\left[A_{1}, A_{2}=0\right]=(1 / 2)(1+\cos \phi),
$$

where we set $\alpha=0$ for simplicity. The partition function is real and its amplitude is zero for $\phi=\pi \times$ integer. This background configuration is fairly singular, and cannot be described by the topological effective action. The situation is similar to the response effective action of the (integer) quantum Hall effect; in the presence of the Chern-Simons term, the response partition function vanishes when one introduces a monopole. (See Ref. [43], for example).

Second, while we have been discussing the free fermion model, the topological response functional (26) can be also derived for more generic models. Consider the Floquet models introduced and discussed in Refs. [23,44]. These models consist of swap operators, acting on each link. As an example, we follow Ref. [44]. The model consists of hard-core bosons living on a square lattice. For each link, we define a SWAP operator,

$$
S_{r, r^{\prime}}\left|n_{r}, n_{r^{\prime}}\right\rangle=\left|n_{r^{\prime}}, n_{r}\right\rangle,
$$

where $n_{r}=0,1$ is the occupation number of hard core bosons at site $r . S_{r, r^{\prime}}$ can be given explicitly as

$$
S_{r, r^{\prime}}=1+b_{r}^{\dagger} b_{r^{\prime}}+b_{r^{\prime}}^{\dagger} b_{r}-n_{r}-n_{r^{\prime}}+2 n_{r} n_{r^{\prime}} .
$$

Combing these SWAP operators, $U_{j}=\prod_{r \in A} S_{r, r+b_{j}}$, the total Floquet drive is given by $U_{F}=U_{4} U_{3} U_{2} U_{1}$. In the absence of boundaries, one can readily check that $U$ is the identity operator,

$$
\left\langle\{n\}\left|U_{F}\right|\left\{n^{\prime}\right\}\right\rangle=\delta_{\{n\},\left\{n^{\prime}\right\}}=\prod_{r} \delta_{n_{r}, n_{r}^{\prime}} .
$$

The background $U(1)$ gauge field can be introduced by replacing $b_{r}^{\dagger} b_{r^{\prime}} \rightarrow b_{r}^{\dagger} g_{r^{\prime}} b_{r^{\prime}}$ in $S_{r r^{\prime}}$ where $g_{r r^{\prime}}=g_{r^{\prime} r}^{*}$ and $g_{r r^{\prime}}=e^{i A_{r r^{\prime}}} \in U(1)$. One can check easily $S_{r r^{\prime}}(A)\left|n_{r}, n_{r^{\prime}}\right\rangle=$ $g_{r r^{\prime}}^{n_{r^{\prime}}}\left|n_{r^{\prime}}, n_{r}\right\rangle . U_{F}(A)$ is diagonal in the occupation number basis and given by

$$
\left\langle\{n\}\left|U_{F}(A)\right|\left\{n^{\prime}\right\}\right\rangle=\exp [i I(n, A)] \delta_{\{n\},\left\{n^{\prime}\right\}} .
$$

Here, for a fixed configuration $\{n\}, e^{i I(n, A)}$ can be written as

$$
e^{i I(n, A)}=\prod_{r}^{n_{r}=1} e^{i B_{r}}=\prod_{r} e^{i B_{r} n_{r}}=e^{i \sum_{r} B_{r} n_{r}},
$$

where the product $\prod_{r}^{n_{r}=1}$ is over all $r$ where a particle is present, $n_{r}=1$. It is then straightforward to see that the topological response functional is given by (26).

Third, while we have focused for the Floquet unitary at $t_{1}-t_{0}=$ (integer) $\times T$, we can monitor the time evolution of the partition function $Z\left[t_{1}, t_{0} ; A_{1}, A_{2}\right]$ numerically for arbitrary $t_{0,1}$ and for a given static gauge field configuration. In Fig. 2, 
(a)

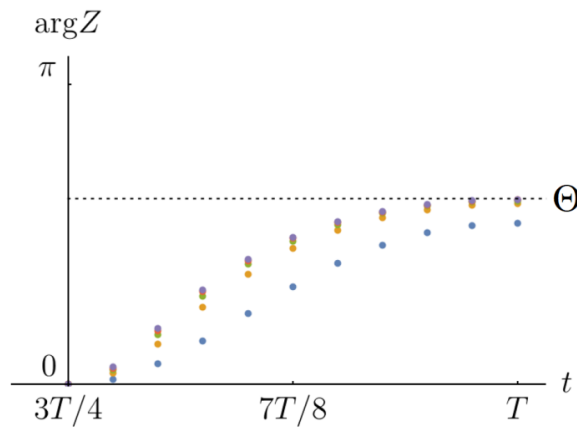

(b)

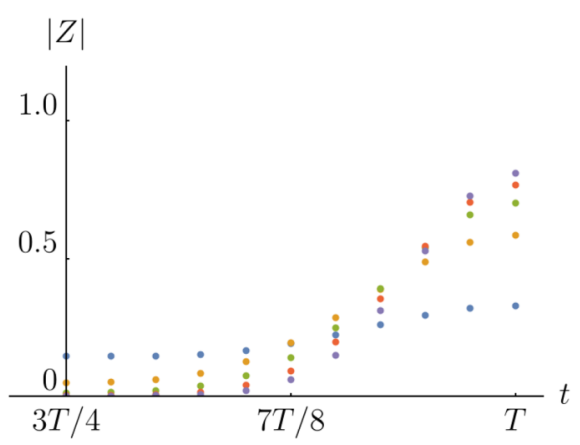

FIG. 2. The time evolution of $Z\left[A_{1}, A_{2}\right]$ for the static gauge field configuration (36) for $3 T / 4<t<T$ for $L_{x}=L_{y}=4,8,12,16,20$ with $\alpha=0.8$. Here $t=t_{1}-t_{0}$, with $t_{0}=-T / 2$. The dotted line represents $\Theta(\alpha=0.8)=1.94795$.

$Z\left[t_{1}, t_{0}=-T / 2 ; A_{1}, A_{2}\right]$ is plotted as a function of $t_{1}$ for the background field configuration $A_{2 i}=0$ and

$$
\begin{aligned}
& A_{1 x}(x, y)=0, \\
& A_{1 y}(x, y)= \begin{cases}0, & y=1, \ldots, L_{y}-1, \\
\frac{2 \pi x}{L_{x}}, & y=L_{y} .\end{cases}
\end{aligned}
$$

In this configuration, the magnetic flux is inserted through plaquette located on a row at $y=L_{y}$. The total flux is $2 \pi$. We see that the amplitude $\left|Z\left[A_{1}, A_{2}\right]\right|$ approaches to $\sim 1$ as $t_{1} \rightarrow T / 2$. On the other hand, away from $t_{1}=T / 2$, the amplitude $\left|Z\left[A_{1}, A_{2}\right]\right|$ can be very small (nearly zero); in these time regions, $Z\left[A_{1}, A_{2}\right]$ seems not to be topological in nature. In addition, as $t_{1} \rightarrow T / 2, \arg Z \rightarrow \Theta(\alpha)$.

\section{B. Separating bulk and boundary actions}

We now move on to discuss the chiral Floquet drive in the presence of open boundary conditions. Let us first recall that, in the absence of boundaries, and with background gauge field, the single-particle unitary $\mathcal{U}_{F}(A)$ is diagonal in the occupation number basis, with the diagonal elements depending on the background $A$. Let us now make a boundary by removing some links. While the bulk part of $\mathcal{U}_{F}(A)$ continues to be diagonal, the boundary part is not, as after one period the location of a particle on the boundary is shifted, see Fig. 3. We can then decompose $\mathcal{U}_{F}(A)$ as

$$
\mathcal{U}_{F}(A)=\mathcal{U}_{\text {bulk }}(A) \oplus \mathcal{U}_{\text {bdry }}(A)
$$

(a)

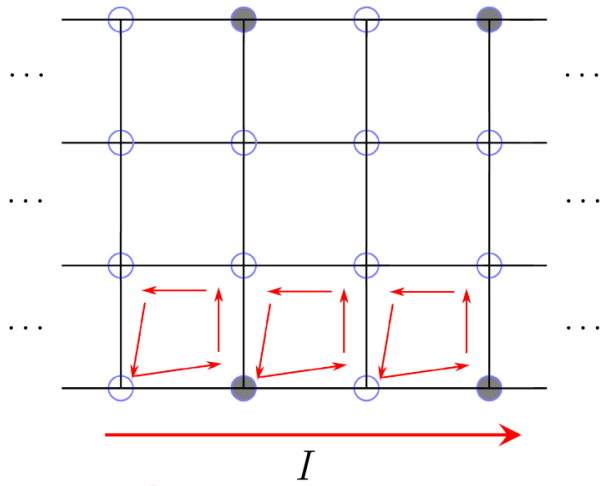

(b)

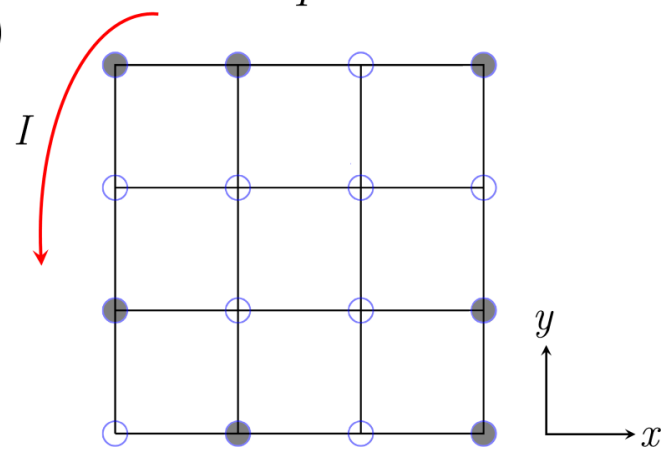

FIG. 3. Chiral Floquet drive with open boundary. (a) Cylindrical geometry with open (periodic) boundary condition in $y(x)$ direction and (b) "Disc" geometry with open boundary condition in both $x$ and $y$ directions. Shaded (unshaded) sites belong to the boundary (bulk) Hilbert space.

where $\mathcal{U}_{\text {bulk }}$ and $\mathcal{U}_{\text {bdry }}$ are supported by two spaces orthogonal to each other; we will refer them as the bulk and boundary Hilbert spaces. For our current model, the boundary Hilbert space consists of a subset of sites living on the boundary, as in Fig. 3. Correspondingly, the many-body Floquet unitary factorizes, $U_{F}=U_{\text {bulk }} \otimes U_{\text {bdry }}$, leading to the factorization of the generating functional:

$$
\begin{aligned}
e^{i W\left[A_{1}, A_{2}\right]}= & \operatorname{Tr}\left[U\left(A_{1}\right) \rho_{0} U^{\dagger}\left(A_{2}\right)\right] \\
= & \operatorname{Tr}\left[U_{\text {bulk }}\left(A_{1}\right) \rho_{0, \text { bulk }} U_{\text {bulk }}^{\dagger}\left(A_{2}\right)\right] \\
& \times \operatorname{Tr}\left[U_{\text {bdry }}\left(A_{1}\right) \rho_{0, \text { bdry }} U_{\text {bdry }}^{\dagger}\left(A_{2}\right)\right] \\
= & e^{i W_{\text {bulk }}\left[A_{1}, A_{2}\right]} e^{i W_{\text {brry }}\left[A_{1}, A_{2}\right]},
\end{aligned}
$$

where we also split the initial density matrix into bulk and boundary parts: $\rho_{0}=\rho_{0, \text { bulk }} \otimes \rho_{0, \text { bdry }}$.

The bulk effective functional $W_{\text {bulk }}\left[A_{1}, A_{2}\right]$ can be computed in the same way as the torus case, and is essentially given by (26), where now in the product $\prod_{r}$ we simply remove sites which belong to the boundary Hilbert space. Taking the continuum limit,

$$
W_{\text {bulk }}\left[A_{1}, A_{2}\right]=\frac{\Theta(\alpha)}{2 \pi} \int \frac{d t}{T} \int_{\text {bulk }} d^{2} r\left[B_{1}(r)-B_{2}(r)\right] .
$$

We also note that

$$
\frac{Z_{\text {bulk }}\left[-A_{1},-A_{2},-\alpha\right]}{Z_{\text {bulk }}\left[A_{1}, A_{2}, \alpha\right]}=e^{i \int \frac{d t}{T} \sum_{r}^{\text {bulk }}\left(B_{1 r}-B_{2 r}\right)} \neq 1,
$$


since the total flux $\sum_{r}^{\text {bulk }} B_{r}$ on an open manifold is not subject to the quantization condition; particle-hole symmetry is broken.

The generating functional for the boundary unitary $W_{\text {bdry }}$ can also be evaluated explicitly:

$$
\begin{aligned}
Z_{\mathrm{bdry}}\left[A_{1}, A_{2}, \alpha\right]= & \frac{1}{\left(2 \cosh \frac{\alpha}{2}\right)^{N_{\mathrm{bdry}}}} \\
& \times \prod_{x}\left\{e^{-\frac{\alpha}{2}}+e^{\frac{\alpha}{2}} e^{i \sum_{x^{\prime}=x}^{x+2 \kappa}\left[A_{1}\left(x^{\prime}\right)-A_{2}\left(x^{\prime}\right)\right]}\right\} .
\end{aligned}
$$

Here we chose the geometry ( $a$ ) in Fig. 3 and populated only one of the boundaries, $x$ labels the sites on the boundary, $A(x)=A_{x, x+1}$ for both $A_{1}(x)$ and $A_{2}(x)$, and $N_{\text {bdry }}$ is the total number of boundary sites. Note that in contrast to the bulk unitary the boundary unitary is not gapped (many-body localized), $U_{\mathrm{bdry}}(A=0)$ is not identity. We can also verify

$$
\frac{Z_{\text {bdry }}\left[-A_{1},-A_{2},-\alpha\right]}{Z_{\text {bdry }}\left[A_{1}, A_{2}, \alpha\right]}=e^{-i \int \frac{d t}{T} \sum_{\text {bdry }}\left(A_{1}-A_{2}\right)} \neq 1,
$$

where $\sum_{\text {bdry }}$ represents the sum taken over links on the boundary region (an analog of a 1D line integral $\oint$ along the boundary). The "Wilson loop" $e^{-i \int \frac{d t}{T} \sum_{\text {dery }}\left(A_{1}-A_{2}\right)}$ is not subject to quantization condition, and hence particle-hole symmetry is broken, as in the bulk. On the one hand, when the bulk and boundary generating functionals are combined, the total generating functional respects the particle-hole symmetry, $Z\left[-A_{1},-A_{2},-\alpha\right] / Z\left[A_{1}, A_{2}, \alpha\right]=1$. The situation is similar for the trace of the single unitary operator $\operatorname{Tr}\left[U_{\text {bdry }}(A)\right]$ (which is not the Schwinger-Keldysh trace), which takes a simple form and is given by

$$
\operatorname{Tr}\left[U_{\mathrm{bdry}}(A)\right]=e^{-L_{x} \frac{\alpha}{2}}+e^{+L_{x} \frac{\alpha}{2}} e^{i \sum_{\text {brry }} A},
$$

where $L_{x}$ is the total number of sites in the boundary Hilbert space. The trace (43) does not preserve particle-hole symmetry, while it enjoys the large $U(1)$ gauge invariance. On the other hand, by adding (multiplying) a counterterm, $e^{-(i / 2) \int \frac{d t}{T} \sum_{\text {bdry }} \operatorname{Tr}\left[U_{\text {bdry }}(A)\right]}$ is particle-hole symmetric, but not invariant under large $U(1)$ gauge transformations. The situation is completely analogous to the well-known mixed anomaly (a conflict between particle-hole and $U(1)$ symmetry) in $(0+1)$ dimensional field theory [45]. This is consistent with the fact that the boundary unitary realizes a single chiral (Weyl) fermion; the single-particle boundary unitary in momentum space is given simply by $\mathcal{U}_{\text {bdry }}(A)=\exp i k_{x}$ where $k_{x}$ is singleparticle momentum along the boundary.

Finally, note that the bulk response (39) has the same form as that of the closed system in Eq. (29). This is expected as the system is localized.

\section{Strip invariant and magnetization}

Let us introduce the Schwinger-Keldysh trace on a strip:

$$
e^{i W_{\text {strip }}\left[A_{1}, A_{2}\right]}=\operatorname{Tr}\left[U\left(A_{1}\right) \rho_{0} P_{\text {strip }} U\left(A_{2}\right)\right],
$$

where $P_{\text {strip }}$ is the projector onto sites contained in the strip shown in Fig. 4. Since the size of the strip is large compared to the localization length, we can take the continuum limit as

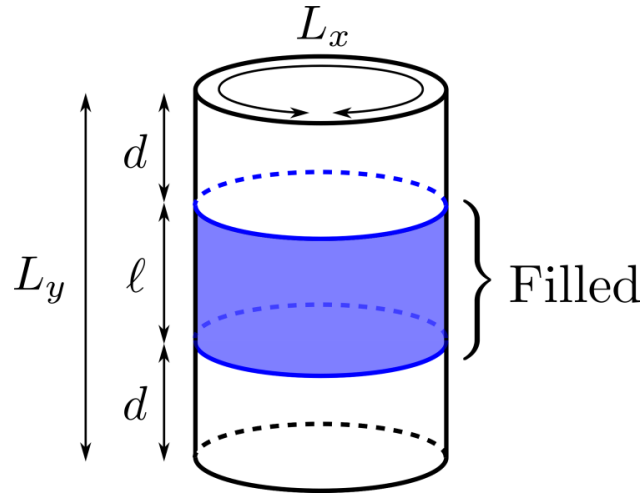

FIG. 4. Representation of the initial state on the cylinder. Sites are populated within the strip indicated in purple. The strip has length $L_{x}$ in the $x$ direction (i.e., it wraps the cylinder), thickness $\ell$ in the $y$ direction, and distance $d$ from the boundaries of the cylinder, with $\ell, d \gg \xi$, where $\xi$ is the localization length.

in the previous section, giving

$$
W_{\text {strip }}\left[A_{1}, A_{2}\right]=\frac{\Theta(\alpha)}{2 \pi} \int \frac{d t}{T} \int_{\text {strip }} d^{2} r\left[B_{1}(r)-B_{2}(r)\right],
$$

which has a similar form as (39), but the spatial integration is now taken over the strip. Despite (39) and (45) look formally identical, it is crucial to note that $\theta=\Theta(\alpha=0)$, as defined in (45), is completely unambiguous and is thus, upon proof of its quantization, $\mathbb{Z}$-valued. Indeed, $\theta$ in (39) could be shifted by multiples of $2 \pi$ by adding boundary contributions to the system, thus making the integer part of $\frac{\theta}{2 \pi}$ a purely boundary quantity [27]. However, the strip invariant (45) is defined on a strip located far away from the boundary. To shift $\theta$ we would need a topological defect in the bulk. ${ }^{7}$ We conclude that $\theta$ is perfectly well defined as a bulk quantity and its value is completely unambiguous. This important point leads us to give (45) a different name, and we shall call it strip invariant.

Now since the magnetic flux can have a continuous value, we can differentiate $W_{\text {strip }}[B, 0]$ with respect to a uniform background magnetic field $B$ and directly relate our response to magnetization. Indeed,

$$
\begin{aligned}
& \left.\frac{\partial}{\partial B} e^{i W_{\text {strip }}[B, 0]}\right|_{B=0} \\
& \quad=\operatorname{Tr}\left[\rho_{0} U^{\dagger}(B=0) \frac{\partial}{\partial B} U(B)\right] \\
& \quad=-i \int_{-\kappa T}^{\kappa T} d t \operatorname{Tr}\left[\rho_{0} U^{\dagger}(t,-\kappa T) \frac{\partial H(t)}{\partial B} U(t,-\kappa T)\right] \\
& \quad=-i \int_{-\kappa T}^{\kappa T} d t \operatorname{Tr}\left[\rho_{0} U^{\dagger}(t,-\kappa T) M U(t,-\kappa T)\right],
\end{aligned}
$$

where we suppressed the subscript bulk from various quantities for simplicity, $\kappa$ is a half-integer, and we used

$$
\begin{aligned}
& \left.U^{\dagger}(\kappa T,-\kappa T) \frac{\partial}{\partial B} U(\kappa T,-\kappa T ; B)\right|_{B=0} \\
& \quad=-i \int_{-\kappa T}^{\kappa T} d t U^{\dagger}(t,-\kappa T) \frac{\partial H(t, B)}{\partial B} U(t,-\kappa T),
\end{aligned}
$$

\footnotetext{
${ }^{7}$ In particular, by changing the thickness $\ell$ of the strip $\theta$ would have a jump. We shall not consider this situation in this work.
} 
and where we identify $M \equiv-\partial H / \partial B$ as the magnetization operator. We are then led to introduce

$$
m_{\alpha}=\left.\frac{i}{2 \kappa T L_{x} L_{y}} \frac{\partial}{\partial B} e^{i W_{\text {strip }}[B, 0]}\right|_{B=0},
$$

where the factor of $2 \kappa T$ is the total length of the time integral, and $L_{x} L_{y}$ is the area of the bulk. We naturally view $m_{\alpha}$ as the magnetization averaged over time and space. This quantity was introduced in Ref. [22] for the single-particle "infinite temperature" state. Using the bulk generating functional worked out in (39), we then find

$$
m_{\alpha}=-\frac{\Theta(\alpha)}{2 \pi T},
$$

so that, for $\alpha=0$, the averaged magnetization is halfquantized. In Ref. [22] it was found that the averaged magnetization is quantized. The relative factor of $1 / 2$ is in that we are considering the sum over states with arbitrary particle numbers.

If we focus on the $N$-particle sector of the Hilbert space, then we have the integral quantization of the averaged magnetization. Indeed, explicit evaluation of the generating functional restricted to the $N$-particle sector $Z_{N}\left[A_{1}, A_{2}\right]$ in (25) (evaluated inside the strip of Fig. 4) gives

$$
-i \log Z_{N, \text { bulk }}\left[A_{1}, A_{2}\right]=\frac{2 \theta}{2 \pi} \int \frac{d t}{T} \int d^{2} r\left[B_{1}(r)-B_{2}(r)\right],
$$

for smooth background gauge fields. Note the relative factor of 2 as compared to (39). Using again Eq. (46), where this time $\rho_{0}$ is the density matrix supported on the bulk and restricted to particle number $N$, gives

$$
\frac{\partial}{\partial B} Z_{N, \text { bulk }}[A, 0]=-i \int_{-\kappa T}^{\kappa T} d t \operatorname{Tr}^{\prime} M(t),
$$

where $\operatorname{Tr}^{\prime}$ is the $N$-particle trace taken over the bulk sites. This then gives the time-averaged magnetization per unit area as

$$
\frac{1}{2 \kappa L_{x} \ell} \int_{-\kappa T}^{\kappa T} d t \operatorname{Tr}^{\prime} M(t)=\frac{\theta}{\pi} .
$$

\section{Stability under deformations}

We shall now show that $\Theta(\alpha)$ must be independent of continuous deformations of the Hamiltonian, as far as the system is localized. In any geometry, such as the torus described in Sec. III A or the strip of Fig. 4, consider smoothly deforming the Hamiltonian inside two regions $I$ and $I I$ whose size and distance is much larger than the localization length, and denote by $H_{I}(t), H_{I I}(t)$ the Hamiltonian in region $I, I I$, respectively. Further, assume that the length scale of deformation from $H_{I}(t)$ to $H_{I I}(t)$ is much shorter than the scale of variation of the gauge field $A_{i}$. The response at first derivative order must then be

$$
W\left[A_{a}\right]=\frac{1}{2 \pi} \int \frac{d t}{T} \int d^{2} r \Theta(\alpha, r) \varepsilon^{i j} \partial_{i} A_{a j}(r),
$$

where $\Theta(\alpha, r)$ approaches the value $\Theta_{I}(\alpha), \Theta_{I I}(\alpha)$ in region $I, I I$, respectively, and the chemical potential $\alpha$ is constant everywhere. One can obtain (53) using the same effective field theory approach that led to (17), although in the current setup there is no translation invariance, and thus $\Theta$ is allowed to depend on position. We again emphasize that the approach leading to the local response action (53) [or (17)] relies on the system being localized. Varying the generating functional with respect to $A_{i}(r)$ gives the time-averaged expectation value of the current,

$$
\begin{aligned}
& \left.\frac{\delta e^{i W[A]}}{\delta A_{i}(r)}\right|_{A=0} \\
& =-i \int d t \operatorname{Tr}\left[\rho_{0} U^{\dagger}(t,-\kappa T) \frac{\partial H(t)}{\partial A_{i}(r)} U(t,-\kappa T)\right] \\
& =-i \int d t \operatorname{Tr}\left[\rho_{0} J^{i}(r, t)\right] \equiv-i \bar{J}^{i}(r),
\end{aligned}
$$

where we used steps similar to those around Eq. (46). Plugging in the functional (53) gives

$$
\bar{J}^{i}(r)=-\frac{1}{2 \pi} \int \frac{d t}{T} \varepsilon^{i j} \partial_{j} \Theta(r) .
$$

Due to localization this current should vanish, as far as $r$ is sufficiently far from any boundaries, such as the boundary of the strip in Fig. 4, or the boundary of the cylinder itself. We now show why this is the case for a model of the form $H(t)=$ $H_{0}(t, A)+H_{\text {int }}(t)$, where $H_{0}$ is the chiral Floquet Hamiltonian in (18) and (19), and $H_{\text {int }}(t)$ is a generic interaction term which does not depend on $A_{i}$ and has a generic time dependence, i.e., it does not have to be periodic: Any $H_{\text {int }}(t)$ will be fine as far as the system remains many-body localized. The trace of the current operator for the Hamiltonian $H(t)$ evaluated at $r=\bar{r}$ is

$$
-i \operatorname{Tr}\left[\rho_{0} J^{i}(\bar{r}, t)\right]=\operatorname{Tr}\left[\rho_{0} U^{\dagger}(t)\left(c_{\bar{r}+i}^{\dagger} c_{\bar{r}}-c_{\bar{r}}^{\dagger} c_{\bar{r}+i}\right) U(t)\right] .
$$

If the system is on a closed manifold, where we do not project out any states, then $\rho_{0}$ commutes with $U(t)$ which immediately leads to the vanishing of the trace, $\operatorname{Tr}\left[\rho_{0} U^{\dagger}(t)\left(c_{\bar{r}+i}^{\dagger} c_{\bar{r}}-\right.\right.$ $\left.\left.c_{\bar{r}}^{\dagger} c_{\bar{r}+i}\right) U(t)\right]=\operatorname{Tr}\left[\rho_{0}\left(c_{\bar{r}+i}^{\dagger} c_{\bar{r}}-c_{\bar{r}}^{\dagger} c_{\bar{r}+i}\right)\right]=0$. (See Ref. [46] for a similar discussion). Thus, as far as $W[A]$ is given by the local functional (53), $\Theta(\alpha)$ must be independent of continuous deformations. Let us now consider a system with boundaries, where the region initially populated is located in the deep bulk and sufficiently far from the boundaries, as represented in Fig. 4. Thanks to localization, the populated region remains far from the boundary. Additionally, in this region the current is insensitive to whether the system is open or closed, therefore we conclude that the expectation value of the current still vanishes. In turn, comparing with (55) this implies that $\Theta$ cannot change continuously. Note that in the above proof we did not make any use of the periodicity of the Hamiltonian. Independence on continuous deformations of this topological response is guaranteed solely by localization.

\section{E. Quantization of $\theta$}

We consider the strip invariant (44) for a system populated only within a strip that is located far from the boundary of the lattice, as illustrated in Fig. 4. Since we want to study $\theta=$ $\Theta(0)$, we shall take the chemical potential $\alpha=0$. Applying Eq. (22) we find

$$
e^{i W_{\text {strip }}}=\frac{1}{2^{L \ell}} \operatorname{det}\left[I+P_{\text {strip }} \mathcal{U} \mathcal{U}^{\dagger}(B)\right],
$$


where $P_{\text {strip }}$ is the single-particle projector on the strip, and $\mathcal{U}$ is the single-particle evolution operator. To first order in $B$,

$$
\frac{1}{2^{L \ell}} \operatorname{det}\left[\frac{1}{2} I+P_{\text {strip }} \mathcal{U}^{\dagger} \mathcal{U}(B)\right]=1+\frac{B}{2} \operatorname{tr}\left(P_{\text {strip }} \mathcal{U}^{\dagger} \partial_{B} \mathcal{U}\right)
$$

Then, using (39),

$$
\theta=\left.\frac{2 \pi}{i \kappa L \ell} \partial_{B} e^{i W}\right|_{B=0}=\frac{2 \pi}{i \kappa L \ell} \frac{1}{2} \operatorname{tr}\left(P_{\text {strip }} \mathcal{U}^{\dagger} \partial_{B} \mathcal{U}\right) .
$$

In Appendix $\mathrm{C}$ we show that $\theta$ can be written as

$$
\theta=-\pi W\left[\tilde{\mathcal{U}}_{0}\right]=n \pi,
$$

where $W\left[\tilde{\mathcal{U}}_{0}\right]$ is the winding number introduced in Ref. [21], and $\tilde{\mathcal{U}}_{0}(t)$ is a periodization of the unitary $\mathcal{U}(t)$, defined as

$$
\tilde{\mathcal{U}}_{0}(t)=\left\{\begin{array}{ll}
\mathcal{U}\left(t-\frac{\kappa}{2} T,-\frac{\kappa}{2} T\right) & t \in(0, T) \\
e^{i \mathcal{H}_{F}(t-2 T)} & t \in(T, 2 T)
\end{array},\right.
$$

where $\mathcal{H}_{F}$ is the single-particle Floquet Hamiltonian defined by $\tilde{\mathcal{U}}\left(T-\frac{\kappa}{2} T,-\frac{\kappa}{2} T\right)=e^{-i \mathcal{H}_{F} T}$. The proof of (60) uses techniques introduced in Refs. [21,22] and relies on two important elements. One is that the system localizes, which allows us to perform nontrivial manipulations of the trace on the righthand side of (59) and to bring it in the form of a winding number; the other is that our response is evaluated on a timescale much longer than the period $T$, which allows us to discard the nonperiodic part of $\mathcal{U}(t)$.

\section{F. Numerical tests of stability}

As mentioned in the beginning of this section, the topological chiral Floquet model (18) and (19) is somewhat special or ideal in the sense that its Floquet Hamiltonian is zero, $U_{F}=I$. In this subsection, we shall depart from the ideal model (18) and (19) by adding disorder and perturbations, ${ }^{8}$

$$
H(A)=H_{0}(t, A)+\sum_{r} w_{r} c_{r}^{\dagger} c_{r}+\lambda \sum_{r}(-1)^{\eta_{r}} c_{r}^{\dagger} c_{r},
$$

where $H_{0}(t, A)$ is the Hamiltonian introduced in (18) and (19) coupled to gauge field $A_{i}$; the second term is a disorder potential, where $w_{r}$ are uncorrelated and can take values between $[-W, W]$ with equal probability; and, finally, the third term is a clean potential, where $\eta_{r}=0$ or 1 depending on whether $r$ lies in sublattice $A$ or $B$, respectively. [Note that $H_{0}(t, A)$ is zero for $4 T / 5<t<T$ while the last two terms in (62) are present for all $t$.] In the following, we shall probe numerically the stability of the response introduced above. The disorder term, when sufficiently strong, guarantees localization. On the other hand, what the small $\lambda$ perturbation is expected to do is to induce a finite bandwidth in the quasi energy spectrum, and nonzero Floquet Hamiltonian, $H_{F} \neq 0$ : It can compete with the disorder term. Both of these terms, when sufficiently strong, can drive the system away from the topological phase

\footnotetext{
${ }^{8}$ We have also studied different topological Floquet models, which are translationally invariant, characterized by the nonzero 3D winding number topological invariant (and hence nonzero quantized magnetization), and having nonzero Floquet Hamiltonian $H_{F} \neq 0$. The results will be reported elsewhere.
}
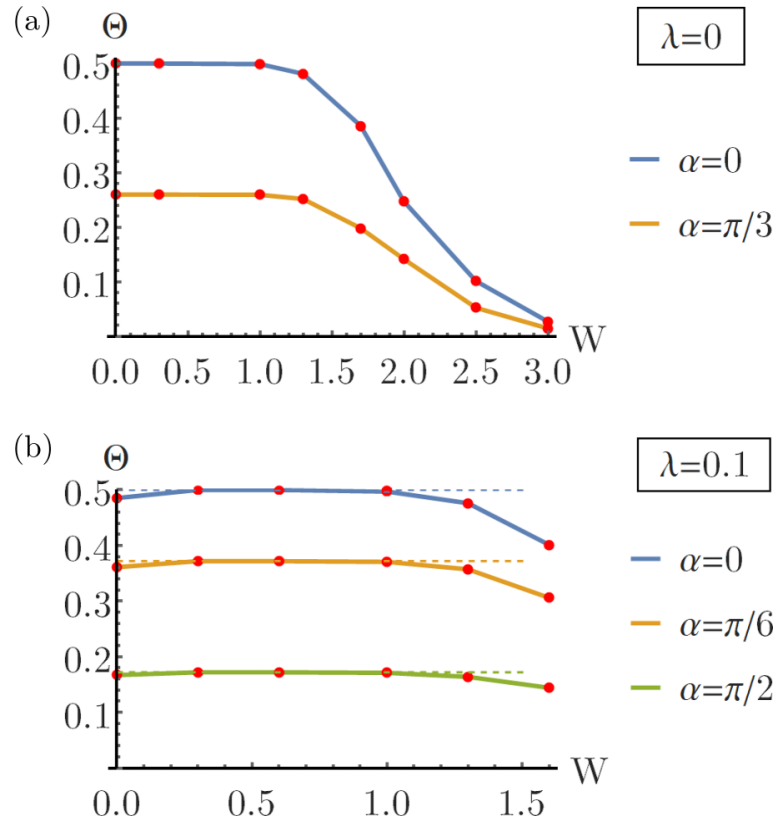

FIG. 5. Plot of $\Theta(\alpha)$ as a function of disorder strength $W$, for various values of $\alpha$ when $\lambda=0$ (a) and $\lambda=0.1$ (b).

with nonzero $\Theta$ by going through a continuous transition. While such transition is interesting, in this paper, we limit our attention to small perturbations to the ideal chiral Floquet drive, and postpone the detailed study of the putative transition to future works.

We study the dependence of $\Theta(\alpha)$ on the disorder strength $W$. To this aim, we simulated the Hamiltonian (62) on a cylindrical lattice of size $L_{x}=20$ and $L_{y}=40$. As initial state, we populated a cylindrical strip of width 16 , so that the distance from the boundaries is sufficiently large compared to the localization length, see Fig. 4. This ensures that we can neglect boundary effects. The generating functional and the Theta angle $\Theta(\alpha)$ are obtained by taking the average of the disorder realizations of the Schwinger-Keldysh trace:

$$
e^{i W_{\text {strip }}\left[A_{1}, A_{2}\right]}=\overline{\operatorname{Tr}\left[U\left(W, A_{1}\right) \rho_{0} P_{\text {strip }} U^{\dagger}\left(W, A_{2}\right)\right]},
$$

where $\cdots$ represents disorder averaging, in the presence of a fixed background field configuration with $\int d A_{1}=2 \pi$ and $\int d A_{2}=0$. In our simulation, we performed 20 disorder realizations. We emphasize that, as mentioned below Eq. (11), we need to evaluate $W\left[A_{1}, A_{2}\right]$ over a very long time in order to isolate the topological terms. One can indeed verify numerically that evaluating $W\left[A_{1}, A_{2}\right]$ over a time which is comparable to the microscopic timescales of the system, $\Theta(\alpha)$ quickly deviates from the unperturbed value as one increases disorder, even if the system is localized. ${ }^{9}$

We first set $\lambda=0$. Figure 5(a) shows the dependence of $\Theta(\alpha)$ on $W$ for different values of $\alpha$. For values of $W$ that are not too large compared to the quasienergy gap of $H_{0}$, $\varepsilon=2 \pi / T=0.8$, one sees the presence of a plateau. At larger

\footnotetext{
${ }^{9}$ Evaluating $W$ over short times is equivalent to having backgrounds $A_{1 i}, A_{2 i}$ that vary fast in time, thus probing quasienergy scales that are characteristic of the microscopic system.
} 


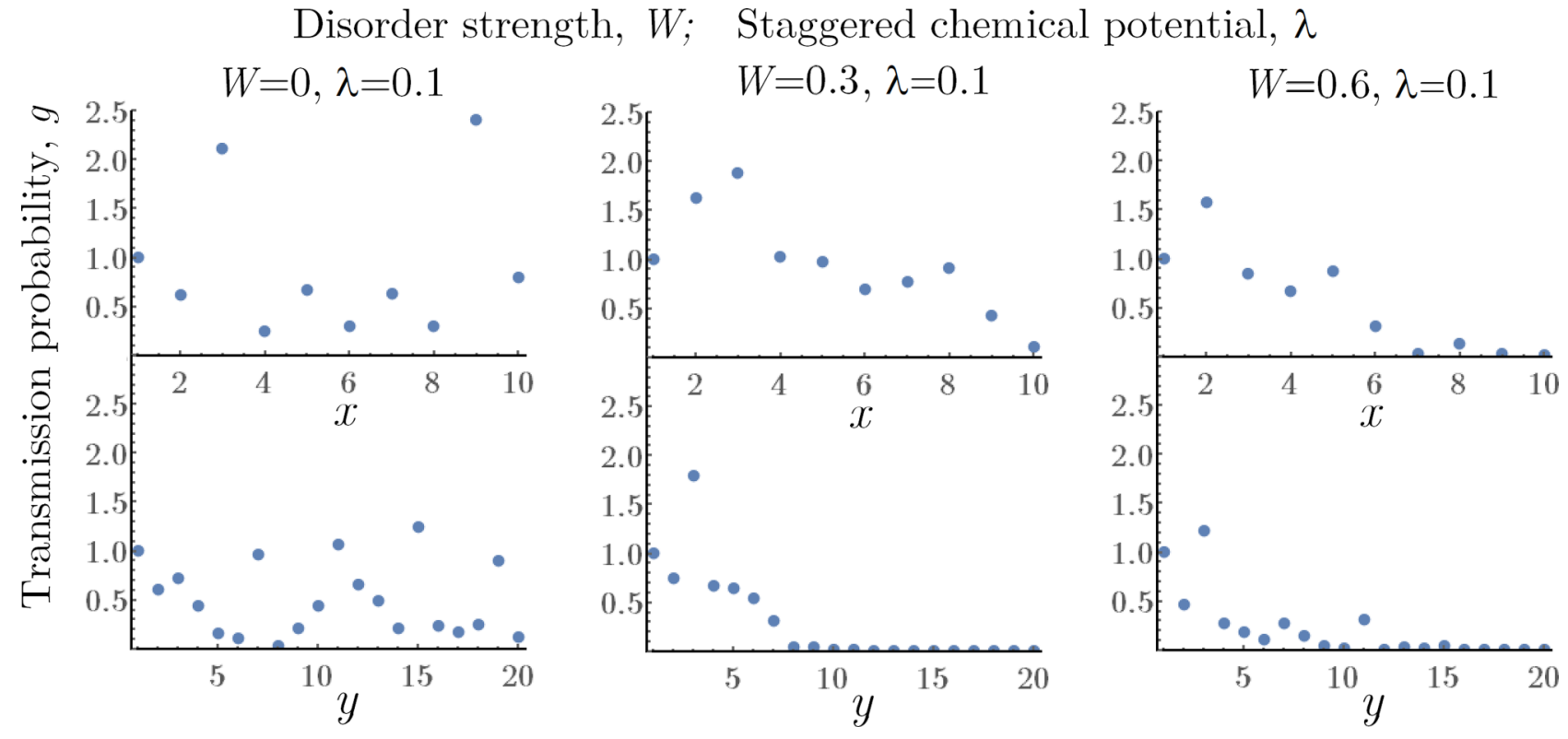

FIG. 6. The plots show the transmission probability $g(r)$ as a function of $r=(x, 0)$ in the first row, and $r=(0, y)$ in the second row. As $W$ becomes larger than $\lambda$, correlations between sites drop exponentially.

values, the disorder seems sufficiently strong to generate a transition to a topologically trivial state. Confirming this requires more accurate numerical simulations, which we leave for future work. As a diagnostics of localization, we considered the normalized transmission probability

$$
g(r)=\max _{w_{r}} \lim _{\kappa \rightarrow \infty}\left|\frac{\left\langle r|\mathcal{U}(\kappa T,-\kappa T ; A=0)| r_{0}\right\rangle}{\left\langle r_{0}|\mathcal{U}(\kappa T,-\kappa T ; A=0)| r_{0}\right\rangle}\right|,
$$

which measures the correlation between a site in the middle of the strip, $r_{0}=\left(0, L_{y} / 2\right)$ and site $r$ after a long-time evolution, and the correlation is maximized over disorder realizations. As plotted in Fig. 6, we see that the system is localized for all values of $W$ near the plateau

Let us now switch on the third term, the clean potential term. Figure 6 shows that the localized regime holds for $\lambda \ll W$, as expected. For $W$ comparable or smaller than $\lambda$, localization is lost and we thus expect to see a deviation of $\Theta(\alpha)$ from the unperturbed value. This indeed happens for $W<0.2-0.3$, as shown in Fig. 5(b), consistently with the delocalization-localization transition which happens around $W=0.2$, as shown in Fig. 6. As $W$ is increased, localization becomes stronger and $\Theta(\alpha)$ is brought back to the unperturbed value. For strong-enough disorder, we again see that $\Theta(\alpha)$ drops to zero.

\section{G. Topological chiral Floquet $p$, q drives}

In this section we apply our response theory to a generalization of chiral Floquet models which is motivated by and related to a class of models introduced in Refs. [24,44]. Those authors found that a class of Floquet systems in two dimensions admits a topological classification by a rational number (Gross-Nesme-Vogts-Werner [47] or chiral unitary index), and characterizes asymmetric quantum information flow at their boundaries. The topological index can be defined without referencing any symmetry, and hence these topological Floquet drives do not require any symmetry for their existence. From the perspective of the SchwingerKeldysh effective field theory approach we are pursuing, one possible way to detect such topological Floquet drives is to introduce a "gravitational" background, and look for a topological term in the gravitational effective action. Here, we instead consider a topological Floquet drive with $U(1)$ symmetry, consisting of excitations with suitably assigned charges, which perform clockwise or counterclockwise chiral motions. We will see that the chiral unitary index is captured by the response we introduced in the earlier part of this section, once we assign charges properly and study the $\alpha$ dependence of the effective functional.

We start again with a square lattice partitioned into two sublattices, precisely as described above Eq. (18). For each site we will now consider a Hilbert space $\mathcal{H}_{\mathrm{p}} \otimes \mathcal{H}_{\mathrm{q}}$, where we further factorize $\mathcal{H}_{\mathrm{p}}=\bigotimes_{i=1}^{\mathrm{r}} \mathcal{H}_{\mathrm{p}_{i}}$ and $\mathcal{H}_{\mathrm{q}}=\bigotimes_{i=1}^{\mathrm{s}} \mathcal{H}_{\mathrm{q}_{i}}$, where $\mathrm{p}_{i}, \mathrm{q}_{i}$ are prime numbers, and $\mathcal{H}_{\mathrm{k}}$ has dimension $\mathrm{k}$. For a given site $r$, we label states in $\mathcal{H}_{\mathrm{k}}$ by their $U(1)$ charge as $\left|r, n_{\mathrm{k}}\right\rangle$ where $n_{\mathrm{k}}=0, \ldots, \mathrm{k}-1$ is the (particle-hole unsymmetrized) particle number. We then consider the following four-step Floquet drive:

$$
\begin{aligned}
U_{F} & =U_{4} U_{3} U_{2} U_{1}, \\
U_{n} & =\prod_{r}\left(\prod_{\mathrm{p}_{i}} U_{n, r}^{\left(\mathrm{p}_{i}\right)}\right)\left(\prod_{\mathrm{q}_{i}} U_{n, r}^{\left(\mathrm{q}_{i}\right)}\right),
\end{aligned}
$$

where the action of these unitaries on a state $\left|r, n_{\mathrm{p}_{i}}\right\rangle$ is

$$
U_{n, r}^{\left(\mathrm{p}_{i}\right)}\left|r, n_{\mathrm{p}_{i}}\right\rangle=e^{i n_{\mathrm{p}_{i}} A_{r, r+b_{n}}}\left|r+b_{n}, n_{\mathrm{p}_{i}}\right\rangle,
$$

and, similarly, the action on a state $\left|r, n_{\mathrm{q}_{i}}\right\rangle$ is

$$
U_{n, r}^{\left(\mathrm{q}_{i}\right)}\left|r, n_{\mathrm{q}_{i}}\right\rangle=e^{i n_{\mathrm{q}_{i}} A_{r, r+b_{5-n}}}\left|r+b_{5-n}, n_{\mathrm{q}_{i}}\right\rangle .
$$

In summary, $U$ swaps the location of p-type particles following counterclockwise rotation as in the chiral Floquet 
model (18) and (19), while it swaps the location of q-type particles following clockwise rotation. This type of evolution was introduced in Refs. [24,44]. In our case, we additionally assign $U(1)$ charges to particles so that our response can directly capture the topology of those models. In Refs. [24,44], the topological classification was demonstrated by deformation arguments, where the deformations involved exchanging subspaces of $\mathcal{H}_{\mathrm{p}}$ of dimension $\mathrm{p}_{i}$ with subspaces of $\mathcal{H}_{\mathrm{q}}$ of dimension $\mathrm{q}_{i}$ whenever $\mathrm{p}_{i}=\mathrm{q}_{i}$. This leads to a topological classification labeled by the factors $\mathrm{p}_{i}$ and $\mathrm{q}_{i}$ which are pairwise coprime, i.e., the classification is labeled by $p / q$. Our assignment of charges has been made so that such deformations. preserve the $U(1)$ symmetry of our Hamiltonian. We can then hope that the response functional $W\left[A_{1}, A_{2}\right]$ will automatically capture the topology property detected and classified by the chiral unitary index. This will turn out to be the case, which illustrates how $W$ furnishes a systematic diagnostic tool for topology. It would be interesting to deal directly with the neutral system, coupling it to a metric rather than a $U(1)$ gauge field. We leave this for future work. (See, however, Sec. IV A for a possible geometric response of topological chiral Floquet drive).

Let us now obtain the generating functional. First, the initial density matrix is $\rho_{0}=e^{\alpha Q} / \operatorname{Tr} e^{\alpha Q}$, with $Q$ the total charge,

$$
Q=\sum_{r}\left(\sum_{i} \tilde{n}_{\mathfrak{p}_{i}, r}+\sum_{j} \tilde{n}_{\mathrm{q}_{j}, r}\right),
$$

where we again used particle-hole symmetrized numbers $\tilde{n}_{\mathrm{k}}$, in the sense that the map $n_{\mathrm{k}} \rightarrow \mathrm{k}-1-n_{\mathrm{k}}$ becomes $\tilde{n}_{\mathrm{k}} \rightarrow$ $-\tilde{n}_{\mathrm{k}}$. One then finds

$$
\operatorname{Tr} e^{\alpha Q}=\prod_{r} \prod_{\mathrm{p}_{i}}\left[\sum_{k=0}^{\mathrm{p}_{i}-1} e^{\left(k-\frac{\mathrm{p}_{i}-1}{2}\right) \alpha}\right] \prod_{\mathrm{q}_{j}}\left[\sum_{k=0}^{\mathrm{q}_{j}-1} e^{\left(k-\frac{\mathrm{q}_{j}-1}{2}\right) \alpha}\right] .
$$

Repeating similar steps as those in the beginning of Sec. III A, we obtain the generating functional

$$
\begin{aligned}
e^{i W\left[A_{a}\right]}= & \frac{1}{\operatorname{Tr} e^{\alpha Q}} \prod_{r} \prod_{\mathrm{p}_{i}}\left[\sum_{k=0}^{\mathrm{p}_{i}-1} e^{\left(k-\frac{\mathrm{p}_{i}-1}{2}\right) \alpha} e^{+i k \int \frac{d t}{T} B_{r}}\right] \\
& \times \prod_{\mathrm{q}_{i}}\left[\sum_{k=0}^{\mathrm{q}_{i}-1} e^{\left(k-\frac{\mathrm{q}_{i}-1}{2}\right) \alpha} e^{-i k \int \frac{d t}{T} B_{r}}\right] .
\end{aligned}
$$

The structure of this generating functional is similar to that of (26), where, at each site $r$, we sum over all possible particle numbers and the corresponding flux collected through the micromotion of each particle around the corresponding plaquette. The continuum limit gives

$$
W\left[A_{a}\right]=\frac{\Theta_{\mathrm{p}, \mathrm{q}}(\alpha)}{2 \pi} \int \frac{d t}{T} \int d^{2} r B_{a}(r),
$$

where

$$
\Theta_{\mathrm{p}, \mathrm{q}}(\alpha)=\theta_{\mathrm{p}, \mathrm{q}}+f_{\mathrm{p}, \mathrm{q}}(\alpha)
$$

with

$$
\begin{aligned}
\theta_{\mathrm{p}, \mathrm{q}}= & \pi \sum_{i=1}^{\mathrm{r}}\left(\mathrm{p}_{i}-1\right)-\pi \sum_{i=1}^{\mathrm{s}}\left(\mathrm{q}_{i}-1\right), \\
f_{\mathrm{p}, \mathrm{q}}(\alpha)= & \pi \sum_{i=1}^{\mathrm{r}}\left(\mathrm{p}_{i} \operatorname{coth} \frac{\mathrm{p}_{i} \alpha}{2}-\operatorname{coth} \frac{\alpha}{2}\right) \\
& -\pi \sum_{i=1}^{\mathrm{s}}\left(\mathrm{q}_{i} \operatorname{coth} \frac{\mathrm{q}_{i} \alpha}{2}-\operatorname{coth} \frac{\alpha}{2}\right) .
\end{aligned}
$$

Notice that if there are common factors $\mathrm{p}_{i}=\mathrm{q}_{i}$, then the corresponding terms will cancel out in $\Theta_{p, q}(\alpha)$, so the continuum limit depends only on factors of the two respective sets $\left\{\mathrm{p}_{i}, i=1, \ldots, \mathrm{r}\right\}$ and $\left\{\mathrm{q}_{i}, i=1, \ldots, \mathrm{s}\right\}$ which are different from each other, i.e., the response exactly depends on $p / q$ ! This is fully consistent with the chiral unitary index, which we now recover as a topological response. Interestingly, one can see that the phase of (70) is also only dependent on p/q. Following the argument of Sec. III F, one then concludes that $\Theta_{p, q}(\alpha)$ is independent of localization-preserving deformations of the system.

\section{MORE ON EFFECTIVE THEORY OF RESPONSE}

In Sec. III, our primary focus was to derive or calculate the Schwinger-Keldysh effective response functional starting from microscopic models such as the 2D chiral Floquet drive. However, one of the advantages of the effective field theory approach is that, based on a few basic principles, one can put constraints on allowed terms in the effective action, and systematically enumerate them, even without knowing microscopic details of the system. In this section, we illustrate the advantage of the effective field theory approach to response by describing two new types of quantized response. We should emphasize that, while the examples below are consistent with the effective field theory of response, we do not yet know whether and how they can be realized microscopically, which we leave to future work.

\section{A. Geometric response}

For the first example, we consider the response to particular geometric deformations. Recall that Floquet systems are invariant under discrete time translation by a period $T$ and that, since we probe the long-time behavior, time translation can be viewed as a continuous symmetry. We now gauge this symmetry and introduce a corresponding gauge field. The gauge symmetry acts on the time coordinate as follows:

$$
t \rightarrow t+f(\vec{r}) .
$$

The corresponding gauge field, which we denote as $a_{i}$, transforms as an Abelian gauge field $\delta a_{i}=-\partial_{i} f(\vec{r})$. The gauge-invariant generating functional is

$$
e^{i W}=\operatorname{Tr}\left[U\left(\infty,-\infty ; a_{1 i}\right) \rho_{0} U^{\dagger}\left(\infty,-\infty ; a_{2 i}\right)\right],
$$

where $\rho_{0}$, up to normalization, is the identity, or the projector on a strip such as that in Fig. 4. Gauge invariance of the generating functional $W$ implies that the current conjugated 
to $a_{i}$ is conserved (in the absence of other external fields):

$$
Q^{i}=\frac{\delta W}{\delta a_{i}}, \quad \partial_{i} Q^{i}=0 .
$$

The current $Q^{i}$ is the (quasi-)energy current since time translation symmetry is responsible for the (quasi-)energy conservation.

To the leading order in derivatives $W$ takes the following form:

$$
W=\int \frac{d t}{T} \int d^{2} r c_{1}\left(\varepsilon^{i j} \partial_{i} a_{1 j}-\varepsilon^{i j} \partial_{i} a_{2 j}\right),
$$

where as before the time integration is done on $t \in$ $(-\kappa T, \kappa T), \kappa$ a half-integer which we shall take to infinity at the end, and where the factor of $1 / T$ has been inserted for convenience. We consider a geometry without boundaries where $a_{i}$ has a nontrivial flux. The spatial slice is assumed to be flat with the periodic boundary conditions, while $a_{i}$ is given by

$$
a_{i}=\omega \epsilon^{i j} r_{j}, \quad \omega=\frac{k T}{L^{2}},
$$

where $T$ and $L$ are defined through the twisted space-time boundary conditions

$$
\begin{gathered}
t \sim t+T, \quad r_{1} \sim r_{1}+L, \\
\left(t, r_{1}, r_{2}\right) \sim\left(t-\omega L\left(r_{1}-r_{2}\right), r_{1}, r_{2}+L\right) .
\end{gathered}
$$

Consistency of the above coordinate identifications implies that $k$ is an integer. ${ }^{10}$ The flux of $a_{i}$ will then be $2 \omega$ which is quantized. The fact that real time is periodic means that we can consistently place on this geometry only systems whose evolution is truly periodic, $U\left(t, t_{0}\right)=U\left(t+T, t_{0}\right)$.

An example of such system is the unperturbed chiral Floquet model of Sec. III. Suppose that the system has time-reversal invariance, in the sense that $H^{T}\left(t, a_{i}\right)=$ $H\left(-t,-a_{i}\right) .{ }^{11}$ Following the reasoning around (29), one then requires

$$
\begin{aligned}
c_{1} \int_{-\kappa T}^{\kappa T} \frac{d t}{T} \int d^{2} r \varepsilon^{i j} \partial_{i} a_{j} & =c_{1} 2 \kappa L^{2} 2 \omega=2 \pi \\
& \Longrightarrow \quad c_{1} \in \frac{\pi}{T} \mathbb{Z},
\end{aligned}
$$

thus leading to quantization of $c_{1}$.

Next we discuss the physics interpretation of $c_{1}$. This coefficient describes the time-averaged "thermodynamic" quantity known as energy magnetization [48-50]. It is defined as the variational derivative $\mathrm{e}^{12}$

$$
m_{E}=\frac{\delta W}{\delta\left(\epsilon^{i j} \partial_{i} a_{j}\right)},
$$

\footnotetext{
${ }^{10}$ Indeed, the composition of the second identification followed by the third one in (79) results in an identification which is equal to the composition of the third followed by the second one, up to a shift of time $t \sim t-\omega L^{2}$.

${ }^{11}$ One could define a slightly more general notion of time-reversal invariance, i.e., $H^{T}\left(t, a_{i}\right)=H\left(t_{0}-t,-a_{i}\right)$. This definition is equivalent to the one in the main text up to translating the definition of the Hamiltonian $H\left(t, a_{i}\right) \rightarrow H^{\prime}\left(t, a_{i}\right) \equiv H\left(t+t_{0} / 2, a_{i}\right)$.

${ }^{12}$ Here we assume that $W$ depends on $a_{i}$ only through its flux.
}

giving

$$
Q^{i}=\varepsilon^{i j} \partial_{j} m_{E},
$$

which justifies the definition. With this definition at hand we find that the energy magnetization takes the form

$$
m_{E}=\frac{c_{1}}{2 \pi T} .
$$

The coefficients $\Theta$ in (17) and $c_{1}$ are completely independent of each other and provide two independent invariants characterizing a topological Floquet phase. Comparing to the quantization of the magnetization we have a relative factor of $2 \pi / T .^{13}$

\section{B. Time-ordering sensitive topology}

We now turn to the second extension of our effective response. So far we have seen response of factorized form $W\left[A_{1}, A_{2}\right]=W_{0}\left[A_{1}\right]-W_{0}\left[A_{2}\right]$, i.e., the two SchwingerKeldysh copies of the background are decoupled, and setting one of them to zero would yield equivalent amount of information. We now show that, at least from the point of view of the effective field theory, this is not always the case. The fact that the two copies can talk to each other gives rise to an additional type of topological terms which are related to time ordering. The most immediate example is the response to a $U$ (1) gauge field in $6+1$ dimensions. At leading derivative order, the most general generating functional is

$$
\begin{aligned}
W= & \frac{1}{4 \pi^{2}} \int \frac{d t}{T} \int d^{6} r \varepsilon^{i j k l p q}\left[3 c_{2} \partial_{i} A_{r j} \partial_{k} A_{r l} \partial_{p} A_{a q}\right. \\
& \left.+\left(c_{3}+c_{2} / 4\right) \partial_{i} A_{a j} \partial_{k} A_{a l} \partial_{p} A_{a q}\right],
\end{aligned}
$$

where $c_{2}, c_{3}$ are constants, and we set the chemical potential to zero for simplicity. Moreover, we conveniently introduced

$$
A_{r i}=\frac{1}{2}\left(A_{1 i}+A_{2 i}\right), \quad A_{a i}=A_{1 i}-A_{2 i} .
$$

The part proportional to $c_{2}$ can be factorized into

$$
c_{2} \varepsilon^{i j k l p q}\left(\partial_{i} A_{1 j} \partial_{k} A_{1 l} \partial_{p} A_{1 q}-\partial_{i} A_{2 j} \partial_{k} A_{2 l} \partial_{p} A_{2 q}\right),
$$

where the two copies of the background are decoupled as before. This means that, if $c_{3}=0, c_{2}$ captures information related to the time average of a time-ordered correlation function. The coefficient $c_{3}$ couples nontrivially $A_{1 i}$ and $A_{2 i}$, and is related to the time average of a nontime-ordered correlation function. To see this more explicitly, let us specialize to the background configuration

$$
\begin{aligned}
& \left(A_{s 1}, A_{s 2}\right)=\frac{B_{s, 12}}{2}\left(-r_{2}, r_{1}\right), \\
& \left(A_{s 3}, A_{s 4}\right)=\frac{B_{s, 34}}{2}\left(-r_{4}, r_{3}\right), \\
& \left(A_{s 5}, A_{s 6}\right)=\frac{B_{s, 56}}{2}\left(-r_{6}, r_{5}\right),
\end{aligned}
$$

\footnotetext{
${ }^{13}$ This comes from that the "charge" of the system with respect to large time translations is $T$, due to the first identification in (79), while in the magnetization case, the $U(1)$ charge is $2 \pi$.
} 
where $s=1,2$ labels the Schwinger-Keldysh copies. Then (85) gives

$$
\left.\frac{\partial^{3} e^{i W}}{\partial B_{1,12} \partial B_{1,34} \partial B_{1,56}}\right|_{B=0}=\frac{3 \kappa L^{6}}{2 \pi^{2}}\left(c_{2}+2 c_{3}\right),
$$

where $L^{6}$ is the volume of the system and $\kappa=\frac{1}{2} \int \frac{d t}{T}$ is a half-integer as usual. Now introduce time-dependent $B_{1,12}(t), B_{1,34}(t), B_{1,56}(t)$. Using (7),

$$
\begin{aligned}
& \left.\frac{\delta^{3} e^{i W}}{\delta B_{1,12}\left(t_{1}\right) \delta B_{1,34}\left(t_{2}\right) \delta B_{1,56}\left(t_{3}\right)}\right|_{B=0} \\
& =\operatorname{Tr}\left[\rho_{0} T\left(M_{12}\left(t_{1}\right) M_{34}\left(t_{2}\right) M_{56}\left(t_{3}\right)\right)\right],
\end{aligned}
$$

where $M_{12}(t)$ is the magnetization operator coupled to $B_{12}$ in the Heisenberg picture, ${ }^{14}$

$$
M_{12}(t)=-U^{\dagger}(t,-\infty)\left[\frac{\partial H(t)}{\partial B_{12}}\right]_{t} U(t,-\infty),
$$

and similarly for $M_{34}(t)$ and $M_{56}(t)$. Note that (89) is the timeintegrated counterpart of (94), so that

$c_{2}+2 c_{3}=\frac{2 \pi^{2}}{3 \kappa L^{6}} \int d t_{1} d t_{2} d t_{3} \operatorname{Tr}\left[\rho_{0} T\left(M_{12}\left(t_{1}\right) M_{34}\left(t_{2}\right) M_{56}\left(t_{3}\right)\right)\right]$,

i.e., $c_{2}+2 c_{3}$ is the time integral of a time-ordered three-point function of magnetization operators. Similarly, one gets

$$
\left.\frac{\partial^{3} e^{i W}}{\partial B_{1,12} \partial B_{1,34} \partial B_{2,56}}\right|_{B=0}=-\frac{3 \kappa L^{6}}{2 \pi^{2}} c_{3} .
$$

and, using (7),

$$
\begin{aligned}
& \left.\frac{\delta^{3} e^{i W}}{\delta B_{1,12}\left(t_{1}\right) \delta B_{1,34}\left(t_{2}\right) \delta B_{2,56}\left(t_{3}\right)}\right|_{B=0} \\
& \quad=\operatorname{Tr}\left[\rho_{0} T\left(M_{12}\left(t_{1}\right) M_{34}\left(t_{2}\right)\right) M_{56}\left(t_{3}\right)\right],
\end{aligned}
$$

and we see that $c_{3}$ is related to the time average of a threepoint function of the same operators as for $c_{2}+2 c_{3}$, but with different time ordering:

$$
c_{3}=-\frac{2 \pi^{2}}{3 \kappa L^{6}} \int d t_{1} d t_{2} d t_{3} \operatorname{Tr}\left[\rho_{0} T\left(M_{12}\left(t_{1}\right) M_{34}\left(t_{2}\right)\right) M_{56}\left(t_{3}\right)\right] .
$$

It would be very interesting to realize microscopic systems that lead to such "time-order sensitive" topology. We end this section by mentioning that, obviously, one can use standard methods of dimensional reduction to reduce the response (85) to lower dimensions.

\footnotetext{
${ }^{14}$ For simplicity of illustration, in Eq. (94) we neglected terms containing higher derivatives of the Hamiltonian with respect to magnetic field, e.g., $\frac{\partial^{2} H(t, B)}{\partial B_{12} \partial B_{34}}$. If the Hamiltonian has appreciable nonlinear dependence on the magnetic field, then the contribution of such terms in (94) may become important.
}

\section{CONCLUSION}

In this paper, we put forward topological response theory for nonequilibrium topological systems using the SchwingerKeldysh formalism. Taking the chiral Floquet drives in two spatial dimensions as an example, we identify topological terms in the Schwinger-Keldysh generating functional in the presence of static background $U(1)$ gauge field. As yet another example, in Appendix A, we discuss the Schwinger-Keldysh generating functional for topological Floquet unitaries constructed from group cohomology [19] with symmetry $G$ in $d$-spatial dimensions. There again, we identify topological response actions which are elements of $H^{d}(G, U(1))$, in agreement with the previous claim [13-15].

The presence of these topological terms in the response actions provides the (many-body) definition of topological Floquet unitaries, and serves as (many-body) topological invariants. We expect that the Schwinger-Keldysh effectivefield-theory approach should work beyond the models studied in this paper, in generic space dimensions and with various kinds of symmetries. Nevertheless, the case studied in this paper, namely, the 2D topological chiral Floquet drive with $U(1)$ symmetry, may be somewhat special in the sense that the quantized topological term is readily related to the physically meaningful response, i.e., quantized magnetization. For topological terms for other symmetries, it may be more difficult or nontrivial to relate them to insightful, physically measurable responses.

Our approach should work even in the absence of symmetry - one may be able to discuss the coupling of Floquet unitaries to a background gravitational field. This may be of particular interest, since there are topological Floquet unitaries without symmetry $[23,24]$. These systems are characterized by asymmetric quantum information flow at their boundaries, and by the quantized edge topological index. It would be interesting if we can capture the topological index by properly introducing (a lattice version of) gravitational background and by the presence of a topological term in the gravitational effective action. (While we postpone the detailed implementation of this to future works, we discuss the possible geometric response of the coupling of 2D Floquet drives in Sec. IV).

There are plenty of open questions, such as an extension of our work to other symmetries, transitions between different Floquet topological phases, applications of our formalism to other nonequilibrium (topological) systems, etc. Among the most pressing issues is to develop a more comprehensive understanding of the structure of the Schwinger-Keldysh effective topological action. For example, we have limited our focus to background field configurations where $A_{i, 1}, A_{i, 2}$ are time independent and $\alpha$ is a constant. The motivation for this is that we can exactly compute the effective action for these choices, but nevertheless, it would be important to study the effective action for generic time and for more generic background configurations.

Studying the Schwinger-Keldysh effective action in the presence of generic background field configurations seems also important to resolve the following puzzle: We identified the theta term in the Schwinger-Keldysh effective topological action for $2 \mathrm{D}$ chiral Floquet drives, which values in $\mathbb{Z}_{2}$ for 
closed spatial manifolds and in the presence of particle-hole symmetry. While the quantization of magnetization can be discussed by using open spatial manifolds, there is a question if the bulk effective action for closed spatial manifolds can fully capture the topological nature of 2D chiral Floquet drives. Also, the theta term is quantized by particle-hole symmetry. While it does exist in the model we looked at, one would expect that particle-hole symmetry may be a special property of the Floquet drive at particular times, but would ultimately be unnecessary for the fundamental topological property of chiral Floquet drives.

Another point to mention is that the Schwinger-Keldysh effective topological actions studied in this paper all have the factorized form, i.e., the effective response partition function factorizes between two Schwinger-Keldysh copies [see also comments below (A23)]. We may speculate that factorized response partition functions describe only the subset of topological Floquet drives, i.e., there may be topological Floquet drives for which the factorization does not take place, and the effective functional is given by a complicated polynominal of $A_{a}$ and $A_{r}$. This may happen in particular in higher dimensions, as discussed in Sec. IV. We leave detailed study of such systems for future works.

Note added. While finalizing the manuscript, Ref. [46] appeared on arXiv, which has some overlap with our work. Another recent study [51] also considers topological response far from equilibrium.

\section{ACKNOWLEDGMENTS}

We thank useful discussion with Erez Berg, Michael Levin, Yuhan Liu, and Hassan Shapourian. This work was supported in part by the National Science Foundation, Grant No. DMR 1455296, and a Simons Investigator Grant from the Simons Foundation. P.G. was supported by a Leo Kadanoff Fellowship.

\section{APPENDIX A: GROUP COHOMOLOGY MODELS}

In this Appendix, we consider topological Floquet drives preserving discrete symmetry $G$. It has been proposed that topological Floquet systems in $d$ spatial dimensions protected by $G$ can be systematically constructed by using the group cohomology [13-16]

$$
H^{d+1}(G \times \mathbb{Z}, U(1))=H^{d+1}(G, U(1)) \times H^{d}(G, U(1)) .
$$

Here $H^{d+1}(G, U(1))$ corresponds to static SPT phases in $d$ spatial dimensions protected by $G$. On the other hand, $H^{d}(G, U(1))$ describes nontrivial topological unitaries specific to Floquet drives. ${ }^{15}$ For example, when $\quad d=1 \quad$ and $\quad G=\mathbb{Z}_{2} \times \mathbb{Z}_{2}, \quad H^{2}(G \times \mathbb{Z}, U(1))=$ $H^{2}(G, U(1)) \times H^{1}(G, U(1))=\mathbb{Z}_{2} \times\left(\mathbb{Z}_{2} \times \mathbb{Z}_{2}\right)$ Here $H^{2}(G, U(1))=\mathbb{Z}_{2}$ corresponds to the classification of static

\footnotetext{
${ }^{15}$ Recall that static SPT phases described by the group cohomology are expected to exhaustive for low spatial dimensions, and hence the group cohomology classifies all SPT phases.
}

SPT phases protected by with $G=\mathbb{Z}_{2} \times \mathbb{Z}_{2}$, which includes the Haldane phase; $H^{1}(G, U(1))=\mathbb{Z}_{2} \times \mathbb{Z}_{2}$ classifies to nontrivial topological unitaries specific to Floquet drives.

$$
\text { 1. } d=1 \text { and } G=\mathbb{Z}_{2}
$$

It is also possible to construct explicit lattice models corresponding to the group cohomology $H^{d}(G, U(1))$. As an example, consider the case of $d=1$ and $G=\mathbb{Z}_{2}$ [13]. We consider a chain with the two-dimensional on-site local Hilbert space spanned by $\{| \pm\rangle\}$, where $| \pm\rangle$ are the eigen state of the Pauli matrix $\sigma^{x}$ with eigenvalues \pm 1 . The $\mathbb{Z}_{2}$ symmetry is generated by $\prod_{j} \sigma_{j}^{x}$, where the product is over all sites in the chain. We consider the Floquet drive of the form:

$$
\begin{aligned}
U(t) & =e^{-i t H}=\prod_{j} e^{+i t \sigma_{j}^{z} \sigma_{j+1}^{z}} \\
& =\prod_{j}\left[\cos (t)+i \sigma_{j}^{z} \sigma_{j+1}^{z} \sin (t)\right],
\end{aligned}
$$

where $H=-\sum_{j} \sigma_{j}^{z} \sigma_{j+1}^{z}$. When $t=\pi / 2$, and with $\mathrm{PBC}$, $U(t)$ is the identity operator, up to a phase factor:

$$
U(\pi / 2)=\prod_{j}\left[i \sigma_{j}^{z} \sigma_{j+1}^{z}\right]=i^{N},
$$

where $N$ is the total number of lattice sites. While trivial with PBC, the unitary $U(t=\pi / 2)$ is nontrivial with open boundary condition.

Following the spirits of the preceding sections, let us now introduce $\mathbb{Z}_{2}$ gauge fields $\alpha_{j, j+1}= \pm 1$ for links on the chain, and consider:

$$
H[\alpha]=-\sum_{j} \sigma_{j}^{z} \alpha_{j, j+1} \sigma_{j+1}^{z} .
$$

Then, our Floquet unitary is

$$
\begin{aligned}
U(t, \alpha) & =\prod_{j} e^{+i t \sigma_{j}^{z} \alpha_{j, j+1} \sigma_{j+1}^{z}} \\
& =\prod_{j}\left[\cos (t)+i \sigma_{j}^{z} \alpha_{j, j+1} \sigma_{j+1}^{z} \sin (t)\right] .
\end{aligned}
$$

When $t=\pi / 2$ and with $\mathrm{PBC}$,

$$
U(t=\pi / 2, \alpha)=i^{N} \prod_{j} \alpha_{j, j+1}=: i^{N} W(\alpha) .
$$

Hence, $U(t=\pi / 2, \alpha)$ is given by the identity multiplied by the Wilson loop for $\mathbb{Z}_{2}$ gauge field $W(\alpha)= \pm 1$. It follows that the Schwinger-Keldysh trace for two Floquet unitaries $U\left(t, \alpha^{\prime}\right)$ and $U(t, \alpha)$ is given by

$$
Z\left[t ; \alpha, \alpha^{\prime}\right]=\operatorname{Tr}\left[e^{-i t H(\alpha)} e^{+i t H\left(\alpha^{\prime}\right)}\right] .
$$

In particular, when $t=\pi / 2$,

$$
Z\left[t=\pi / 2 ; \alpha, \alpha^{\prime}\right]=W\left(\alpha^{\prime}\right) W(\alpha) .
$$

\section{Generic construction}

\section{a. The Dijkgraaf-Witten theory.}

The above construction for $d=1$ and $G=\mathbb{Z}_{2}$ can be readily extended to more generic cases [19]. To describe the generalization, let us briefly recall the basic ingredients in 


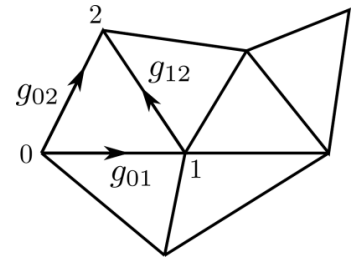

FIG. 7. Triangulation of space time (here for the case of two space-time dimensions) for the Dijkgraaf-Witten model.

the Dijkgraaf-Witten theories [52]. Dijkgraaf and Witten gave a generic construction of (exponentiated) topological actions $\exp \left(i I\left[g, M_{n}\right]\right)$ for discrete gauge theories with gauge group $G$, where $M_{n}$ is $n$-dimensional Euclidean space time, and $\{g\}$ represents a gauge field configuration (see Fig. 7).

The first step of the construction is to triangulate space time in terms of $n$-simplicies ("triangles"), and assign directions (arrows) to each link (e.g., we assign numbers for each vertex in a simplex; for $i<j, \rightarrow$; for $j<i, \leftarrow)$. For each elementary triangle ( $n$-simplex) $\left|\Delta^{n}\right|= \pm 1$ represents the orientation of the simplex with respect to the orientation of space time.

We now assign gauge field $g_{i j} \in G$ to each link. We only consider flat gauge field configurations. For example, when $n=2$, each triangle has three links with three gauge fields $g_{01}, g_{12}$, and $g_{02}$; we impose the flatness condition by $g_{01} g_{12}=g_{02}$, so that two out the three gauge fields are independent. Next, we assign for each $n$-simplex $\Delta^{n}$ a Boltzmann weight $\omega_{n}\left(g_{01}, g_{12}, g_{23}, \cdots\right) \in U(1)$. (For the first entry in $\omega$, we start from the vertex with no incoming edge, etc).
Then, the topological action for a given triangulation is given by

$$
\exp i I\left[g, M_{n}\right]=\prod_{\Delta^{n}} \omega_{n}(\{g\})^{\left|\Delta^{n}\right|} .
$$

As the final step, we demand the action functional to be independent of triangulations of $M_{n}$. This leads to the condition on $\omega_{n}$, the so-called cocycle condition, which is symbolically given by $d \omega_{n}=1$. (We do not write down the definition of $d$ here, but it can be found in the literature). Each solution to this equation gives a topological action $\exp i I\left[g, M_{n}\right]$. Inequivalent solutions to the cocycle condition are classified by the group cohomology $H^{n}(G, U(1))$.

\section{b. SPT partition functions.}

Equation (A9) defines the action functional of the Dijkgraaf-Witten theory (topological gauge theory) on $M_{n}$ with gauge group $G$. Summing over all gauge field configurations $\{g\}$ defines the Dijkgraaf-Witten theory. On the other hand, the action functional $\exp i I\left[g, M_{n}\right]$ itself can be viewed as a response theory of an SPT phase protected by symmetry $G$ [53].

The path integral for the "matter field" can be constructed as follows. We first introduce degrees of freedom living on vertices; let us call them $v_{i} \in G$ (where $v_{i}$ is an element in the group algebra). We introduce $G$-gauge transformations as

$$
v_{i} \rightarrow \alpha_{i} v_{i}, \quad g_{i j} \rightarrow \alpha_{i} g_{i j} \alpha_{j}^{-1} .
$$

Note that combinations $v_{i}^{-1} g_{i j} v_{j}$ are gauge invariant. In some sense, $\left\{v_{i}\right\}$ can be identified as a gauge transformation.

The Dijkgraaf-Witten action is gauge invariant. Hence, we can write

$$
e^{i I\left[g, M_{n}\right]}=\prod_{\Delta^{n}} \omega_{n}\left(v_{0}^{-1} g_{01} v_{1}^{-1}, v_{1}^{-1} g_{12} v_{2}^{-1}, v_{2}^{-1} g_{23} v_{3}^{-1}, \cdots\right)^{\left|\Delta^{n}\right|}=e^{i S\left[g, v, M_{n}\right]} .
$$

Since $\left\{v_{i}\right\}$ is arbitrary, we can sum over $\left\{v_{i}\right\}$,

$$
\begin{aligned}
Z\left[g, M_{n}\right] & =e^{i I\left[g, M_{n}\right]}=\frac{1}{|G|^{N_{v}}} \sum_{\left\{v_{i}\right\}} e^{i S\left[g, v, M_{n}\right]} \\
& =\frac{1}{|G|^{N_{v}}} \sum_{\left\{v_{i}\right\}} \prod_{\Delta^{n}} \omega\left(v_{0}^{-1} g_{01} v_{1}, v_{1}^{-1} g_{12} v_{2}, v_{2}^{-1} g_{23} v_{3}, \cdots\right)^{\left|\Delta^{n}\right|},
\end{aligned}
$$

where $N_{v}$ is the number of vertices. We can then switch off the background field $g$ :

$$
Z\left[M_{n}\right]=\frac{1}{|G|^{N_{v}}} \sum_{\left\{v_{i}\right\}} \prod_{\Delta^{n}} \omega\left(v_{0}^{-1} v_{1}, v_{1}^{-1} v_{2}, v_{2}^{-1} v_{3}, \cdots\right)^{\left|\Delta^{n}\right|} .
$$

This can be considered as a partition function of an SPT phase protected by symmetry $G$. If there is no boundary on $M_{n}$, $Z\left[M_{n}\right]=1$.

It is also convenient to introduce

$$
v\left(g_{0}, g_{1}, g_{2}, g_{3}, \cdots\right) \equiv \omega\left(g_{0}^{-1} g_{1}, g_{1}^{-1} g_{2}, g_{2}^{-1} g_{3}, \cdots\right)
$$

$v$ satisfies (here, we take $n=3$ for simplicity).

$$
\begin{aligned}
v\left(g g_{0}, g g_{1}, g g_{2}, g g_{3}\right)= & v\left(g_{0}, g_{1}, g_{2}, g_{3}\right), v\left(g_{1}, g_{2}, g_{3}, g_{4}\right) v\left(g_{0}, g_{2}, g_{3}, g_{4}\right)^{-1} v\left(g_{0}, g_{1}, g_{3}, g_{4}\right) \\
& \times v\left(g_{0}, g_{1}, g_{2}, g_{4}\right)^{-1} v\left(g_{0}, g_{1}, g_{2}, g_{3}\right)=1 .
\end{aligned}
$$


Conversely, when these conditions are satisfied by $\nu$, one can construct a group cocycle $\omega$ by

$$
\omega\left(g_{1}, g_{2}, g_{3}\right)=v\left(1, g_{1}, g_{1} g_{2}, g_{1} g_{2} g_{3}\right) \text {. }
$$

Using $v$, the partition function can be written as

$$
Z\left[M_{n}\right]=\frac{1}{|G|^{N_{v}}} \sum_{\{v\}} \prod_{\Delta^{n}} v\left(v_{0}, v_{1}, v_{2}, v_{3}, \cdots\right)^{\left|\Delta^{n}\right|} .
$$

\section{c. Group cohomology models realizing topological floquet drives.}

Let us now come back to our question on topological Floquet drives. Can we construct an explicit unitary operator with global symmetry $G$ and for a given space dimension $d$, which, upon introducing a background gauge field, and then taking the Schwinger-Keldysh trace, reproduces the response action functional exp $i I\left[g, M_{d}\right]$, or more precisely exp $i I\left[g_{1}, g_{2}, M_{d}\right]$ ? We can actually simply take the SPT path integral (A17) and "turn" it into a topological Floquet drive. Consider a unitary:

$$
U(t=T)=\sum_{\{v\}} \prod_{\Delta^{d}} v\left(v_{0}, v_{1}, v_{2}, \cdots\right)^{\left|\Delta^{d}\right|}|\{v\}\rangle\langle\{v\}|,
$$

which is completely diagonal. One can check easily that $U(t=T)$ is the identity operator since

$$
\begin{aligned}
\left\langle\{v\}|U(t=T)|\left\{v^{\prime}\right\}\right\rangle & =\delta_{\{v\},\left\{v^{\prime}\right\}} \prod_{\Delta^{d}} v\left(v_{0}, v_{1}, v_{2}, \cdots\right)^{\left|\Delta^{d}\right|} \\
& =e^{i S\left[g=0, v, M_{d}\right]} \delta_{\{v\},\left\{v^{\prime}\right\}} \\
& =e^{i I\left[g=0, M_{d}\right]} \delta_{\{v\},\left\{v^{\prime}\right\}}
\end{aligned}
$$

where we recall (A11). We can introduce a background gauge field and consider:

$$
\begin{aligned}
U(t=T, g)= & \sum_{\{v\}} \prod_{\Delta^{d}} \omega\left(v_{0}^{-1} g_{01} v_{1}, v_{1}^{-1} g_{12} v_{2}, \cdots\right)^{\left|\Delta^{d}\right|} \\
& \times|\{v\}\rangle\langle\{v\}| .
\end{aligned}
$$

Recalling (A11) again,

$$
\begin{aligned}
\left\langle\{v\}|U(t=T, g)|\left\{v^{\prime}\right\}\right\rangle & =e^{i\left[g, v, M_{d}\right]} \delta_{\{v\},\left\{v^{\prime}\right\}} \\
& =e^{i\left[g, M_{d}\right]} \delta_{\{v\},\left\{v^{\prime}\right\}},
\end{aligned}
$$

the Schwinger-Keldysh trace

$$
Z\left[t ; g_{1}, g_{2}\right]=\mathcal{N}^{-1} \operatorname{Tr}\left[U\left(t, g_{1}\right) U^{\dagger}\left(t, g_{2}\right)\right]
$$

is given by, when $t=T$, as a product of the groupcohomology partition functions:

$$
Z\left[t=T ; g_{1}, g_{2}\right]=\exp \left(+i I\left[g_{1}, M_{d}\right]-i I\left[g_{2}, M_{d}\right]\right) .
$$

The topological Schwinger-Keldysh response action (A23) is consistent with the general classification (A1) in the sense that the topological term is (A23) is a member of $H^{d}(G, U(1))$. Equation (A23) is also in harmony with (28) [although its microscopic counterpart (26) is more complicated]. We note that in the group cohomology models, the floquet unitary at $t=T$ is given by the identity operator, up to an over all phase factor which is given by $\omega \in H^{d}(G, U(1))$ [see (A21)]. As a consequence, (A23) simply factorizes $Z\left[t=T ; g_{1}, g_{2}\right]=\mathcal{N}^{-1} \operatorname{Tr}\left[U\left(T, g_{1}\right)\right] \operatorname{Tr}\left[U^{\dagger}\left(T, g_{2}\right)\right]$. This is not the case for the 2D the chiral floquet model; the floquet unitary at $t=T$ is diagonal but not proportional to the identity; (26) does not simply factorize. Nevertheless, $\operatorname{Tr}\left[U\left(T, A_{1}\right) U^{\dagger}\left(T, A_{2}\right)\right]$ depends only on the difference, $A_{a}=$ $A_{1}-A_{2}$, and for smooth (long-wave length) configurations, the topological term can still be written in the factorized form (28). The factorization of the response SchwingerKeldysh action has an affinity with the proposed group cohomology classification (A1), in which we do not see any inkling of the Schwinger-Keldysh formalism; at least naively, the group cohomology $H^{d+1}(G \times \mathbb{Z}, U(1))$ is expected to classify the Euclidean path integral without using the Schwinger-Keldysh copies. Nevertheless, the calculations presented here show the factorization of the SchwingerKeldysh action, and the topological terms in each copies, $\operatorname{Tr}\left[U\left(t, g_{1}\right)\right]$ and $\operatorname{Tr}\left[U^{\dagger}\left(t, g_{2}\right)\right]$, are labeled by $H^{d}(G, U(1))$; we thus land on (A1).

\section{APPENDIX B: CHANNEL-STATE MAP APPROACH}

In this Appendix, we introduce an approach based on the so-called channel-state map (the Choi-Jamiołkowski isomorphism), which maps arbitrary unitary operator, acting on a Hilbert space $\mathcal{H}$, to a state living in a bigger (doubled) Hilbert space, $\mathcal{H} \otimes \mathcal{H}^{*}$. In physics context, this has been used in the thermofield double state, and the thermo field dynamics [54]. This channel-state map allows us to "transplant" the approaches to static (topological) states to (topological) unitaries and develop an effective response theory.

As the Schwinger-Keldysh formalism, the thermo field dynamics provides a framework to describe the real-time nonequilibrium dynamics of finite temperature systems. In particular, at equilibrium, the thermo field dynamics and the Schwinger-Keldysh formalism are equivalent. In some sense, the purpose of this section is to "redo" what we have achieved in the main text using the Schwinger-Keldysh formalism by using the thermo field dynamics (channel-state map), although the precise relation between the Schwinger-Keldysh approach and the approach presented here is not entirely clear.

As a by-product of using the channel-state map, we will be able to make a contact with the common trick used, e.g., in Ref. [25] to derive the periodic table of noninteracting Floquet fermion systems (the "Hermitian map"). There, one first artificially doubles the original (single-particle) Hilbert space, and then embeds Floquet unitaries into a Hermitian operator ("Hamiltonian") acting on the doubled Hilbert space. We will provide a point of view in terms of the channel-state map. 


\section{Operator-state map}

The channel-state map (the Choi-Jamiołkowski isomorphism) applies to an arbitrary quantum channel (tracepreserving completely positive map), and maps it to a quantum state (density matrix) in the doubled Hilbert space. In simplest cases, it maps a unitary operator $U$ acting on the Hilbert space $\mathcal{H}$ to a (pure) state in the doubled Hilbert space $\mathcal{H} \otimes \mathcal{H}^{*}:$

$$
|U\rangle\rangle=(I \otimes U)|\Omega\rangle\rangle,
$$

where $|\Omega\rangle\rangle$ is a maximally entangled state

$$
|\Omega\rangle\rangle=(1 / \sqrt{\mathcal{N}}) \sum_{i}|i\rangle|i\rangle^{*}, \quad \mathcal{N} \equiv \operatorname{dim} \mathcal{H}=\operatorname{Tr}_{\mathcal{H}} I .
$$

Essentially the same mapping from an operator to a state is used in the context of the thermofield double state, where a thermal density operator is mapped to a state (thermofield double state) in the doubled Hilbert space. Observe that the overlap of two states corresponding to unitaries $U$ and $U^{\prime}$ is

$$
\left\langle\left\langle U \mid U^{\prime}\right\rangle\right\rangle=(1 / \mathcal{N}) \operatorname{Tr}_{\mathcal{H}}\left[U^{\dagger} U^{\prime}\right],
$$

which can be represented as a Schwinger-Keldysh pathintegral with the infinite temperature thermal state as the initial state.

\section{Fermionic chiral Floquet drive}

Let us now consider a fermionic system described by a set of fermion annihilation or creation operators, $\left\{\hat{\psi}_{a}, \hat{\psi}_{b}^{\dagger}\right\}=\delta_{a b}$. Here $a, b=1, \ldots, N$ and $N$ is the number of independent "orbitals," i.e., the dimension of the single-particle Hilbert space. Following our general discussion, we double the fermion Fock space, $\mathcal{H} \rightarrow \mathcal{H} \otimes \mathcal{H}$, and consider the state $(I \otimes \hat{U})|\Omega\rangle\rangle$ where $|\Omega\rangle\rangle$ is a suitable maximally entangled state in the doubled Hilbert space. For the current example, an appropriate choice of $|\Omega\rangle\rangle$ is given by

$$
\left.|\Omega\rangle\rangle \equiv \prod_{a} \frac{1}{\sqrt{2}}\left[\hat{\psi}_{a A}^{\dagger}+\hat{\psi}_{a B}^{\dagger}\right]|0\rangle\right\rangle,
$$

where we now have two independent sets of fermion annihilation or creation operators, $\left\{\hat{\psi}_{a A}, \hat{\psi}_{a A}^{\dagger}\right\}$ and $\left\{\hat{\psi}_{a B}, \hat{\psi}_{a B}^{\dagger}\right\}$, acting on each copy of the fermion Fock space, $\mathcal{H}_{A}$ and $\mathcal{H}_{B}$. Note that for a given "site" $a$, the state is a equal superposition of states of charge $q$ on $A$ and $-q$ on $B$, where $q= \pm 1 / 2$ is the total particle number measured from half-filling, $\left.\left.\left.\left[\hat{\psi}_{a A}^{\dagger}+\hat{\psi}_{a B}^{\dagger}\right]|0\rangle\right\rangle=|10\rangle\right\rangle+|01\rangle\right\rangle=\sum_{q} \mid q+$ $1 / 2,-q+1 / 2\rangle)$. The state dual to $\hat{U}$ can be constructed accordingly as $|U\rangle\rangle=(I \otimes \hat{U})|\Omega\rangle$.

We will be interested in "short-range correlated states," i.e., all equal time correlation functions: $\left\langle\left\langle U\left|\hat{\Psi}_{i}^{\dagger} \cdots \hat{\Psi}_{j} \cdots\right| U\right\rangle\right\rangle=$ $\left\langle\left\langle\Omega\left|\hat{U}^{\dagger} \hat{\Psi}_{i}^{\dagger} \hat{U} \cdots \hat{U}^{\dagger} \hat{\Psi}_{j} \hat{U} \cdots\right| \Omega\right\rangle\right\rangle$ are local in the sense that they decay exponentially in distances. As far as evolution driven by $U$ is "local" or "nonergodic," as in the case of many-body localized evolution, we expect that $|U\rangle\rangle$ can be treated as a ground state of a gapped system.

The reference state $|\Omega\rangle\rangle$ is a unique ground state of the "parent" Hamiltonian

$$
\hat{K}_{0}=\sum_{a, b}\left(\hat{\psi}_{a A}^{\dagger} \hat{\psi}_{b B}+\text { H.c. }\right)
$$

acting on $\mathcal{H} \otimes \mathcal{H}$. Similarly, $|U\rangle\rangle$ is a unique ground state of

$$
\begin{aligned}
\hat{K} & =(I \otimes \hat{U}) \hat{K}_{0}\left(I \otimes \hat{U}^{\dagger}\right) \\
& =\sum_{a, b}\left(\hat{\psi}_{a A}^{\dagger} \hat{U} \hat{\psi}_{b B} \hat{U}^{\dagger}+\text { H.c. }\right) .
\end{aligned}
$$

Let us have a closer look at of this mapping for the case of a quadratic Hamiltonian $\hat{H}=\sum_{a, b=1}^{N} \hat{\psi}_{a}^{\dagger} \mathcal{H}_{a b} \hat{\psi}_{b}$ and the corresponding unitary evolution operator $\hat{U}$. The manybody unitary operator $\hat{U}$ defines a unitary matrix $U$ through $\hat{U} \hat{\psi}_{a} \hat{U}^{\dagger}=\mathcal{U}_{a b} \hat{\psi}_{b}$. The state $\left.\left.|U\rangle\right\rangle=(I \otimes \hat{U})|\Omega\rangle\right\rangle$ can be explicitly calculated easily:

$$
\left.|U\rangle\rangle=\prod_{a} \frac{1}{\sqrt{2}}\left[\hat{\psi}_{a A}^{\dagger}+\sum_{b} \mathcal{U}_{a b}^{\dagger} \hat{\psi}_{b B}^{\dagger}\right]|0\rangle\right\rangle .
$$

The parent Hamiltonian is

$$
\hat{K}=\sum_{i, j=1}^{2 N} \hat{\Psi}_{i}^{\dagger} \mathcal{K}_{i j} \hat{\Psi}_{j}=\sum_{a, b=1}^{N}\left[\hat{\psi}_{a A}^{\dagger} \mathcal{U}_{a b} \hat{\psi}_{b B}+\hat{\psi}_{b B}^{\dagger} \mathcal{U}_{a b}^{\dagger} \hat{\psi}_{a B}\right]
$$

where $\Psi^{\dagger}, \Psi$, and the $2 N \times 2 N$ matrix $\mathcal{K}$ are given by

$$
\hat{\Psi}^{\dagger}=\left[\hat{\psi}_{A}^{\dagger} \quad \hat{\psi}_{B}^{\dagger}\right], \mathcal{K}=\left[\begin{array}{cc}
0 & \mathcal{U} \\
\mathcal{U}^{\dagger} & 0
\end{array}\right], \hat{\Psi}=\left[\begin{array}{l}
\hat{\psi}_{A} \\
\hat{\psi}_{B}
\end{array}\right] .
$$

Passing from the original (single-particle) unitary matrix $\mathcal{U}$ to the Hermitian matrix $\mathcal{K}$ is the "Hermitian map" used in, e.g., Ref. [25] to derive the periodic table of Floquet topological systems. While the original Hamiltonian $\mathcal{H}$ is a member of symmetry class A (if we do not assume any symmetry), $\mathcal{K}$ is a member of symmetry class AIII: $\mathcal{K}$ is invariant under the following antiunitary transformation (chiral symmetry):

$$
\begin{aligned}
& \hat{S} \hat{\psi}_{a A} \hat{S}^{-1}=\hat{\psi}_{a A}^{\dagger}, \quad \hat{S} \hat{\psi}_{a B} \hat{S}^{-1}=-\hat{\psi}_{a B}^{\dagger}, \\
& \hat{S} \hat{\psi}_{a A}^{\dagger} \hat{S}^{-1}=\hat{\psi}_{a A}, \quad \hat{S} \hat{\psi}_{a B}^{\dagger} \hat{S}^{-1}=-\hat{\psi}_{a B} .
\end{aligned}
$$

This transformation can be considered as a composition of the modular conjugation operator (tilde conjugation operator) in the Tomita-Takesaki theory (the thermofield double theory), and the swap operation $\hat{\psi}_{A} \leftrightarrow \hat{\psi}_{B}$.

\section{Building effective response field theories by dimensional reduction}

Note that the spectrum of $K$ is gappled and completely "flat": Its eigenvalues are all either \pm 1 . Any $K$ of this form can be obtained from a more "physical" Hamiltonian preserving chiral symmetry and having a energy gap by spectral flattening [55]. As $|U\rangle\rangle$ is realized as a unique ground state of a gapped Hamiltonian $\hat{K}$, its topological properties can be studied and classified by using the techniques of static (symmetry-protected) topological phases.

With the help of the operator-state map, and assuming the presence of reasonable parent Hamiltonians $\hat{K}$, we now proceed to develop effective response field theories. We henceforth resurrect the so-far neglected time dependence in the unitaries, $\hat{U}(t)$, and work with periodic unitaries, $\hat{U}(t+$ $T)=\hat{U}(t)$.

Following the recipe of deriving effective response field theories for static topological phases, we introduce a 
imaginary-time space-time path integral of type (2). Naively, this would introduce yet another time than the real time $t$, which simply enters in the path integral as a parameter; For a Floquet system living on physical $(d+1)$-space-time dimensions, we have $(d+2)$-dimensional space time. As we will see, this issue can be naturally solved if we make a contact with the theory of adiabatic quantum pump, a typical example of which is the Thouless pump in $(1+1)$-dimensional system. The topological properties of Floquet unitary operators in $(d+1)$-dimensions, may be related to $(d+2)$-dimensional topological phases. The response field theory of the latter can be dimensionally reduced to describe the target $(d+$ $1)$-dimensional physics. This means that we are effectively considering the adiabatic evolution of Floquet unitaries $U(t)$ as a function of $t$, while the time evolution of physical states by Floquet unitaries are not adiabatic in general.

Observe also that if we start from systems with nosymmetry (class A), then mapping unitaries to states by the channel-state map transforms the symmetry class from A to AIII by working with the doubled Hilbert space. This is in a perfect harmony with the above dimensional shift $(d+1) \rightarrow$ $(d+2)$, and with the Bott periodicity.

Now, following the recipe of deriving effective response field theories for static topological phases, we introduce a background $U(1)$ gauge field $V=V_{\mu} d x^{\mu}$. This in principle has nothing to do with physical electromagnetic $U$ (1) gauge field $A=A_{\mu} d x^{\mu}$, as it is introduced in the doubled Hilbert space (see the comments below, though). By integrating over the matter field, we would then arrive at the effective response theory. Since we are in $(3+1) d$, and since our Hamiltonian $K$ belongs to class AIII, the topological part of the resulting effective action is given by the axion term:

$$
W_{\text {eff }}[V]=\frac{\theta}{8 \pi^{2}} \int d u d^{3} x \varepsilon^{\mu \nu \kappa \lambda} \partial_{\mu} V_{\nu} \partial_{\kappa} V_{\lambda},
$$

where $u$ is the fictitious imaginary time, which is analytically continued to the Lorentz signature in Eq. (B11). The $\theta$ angle here is pinned to quantized values, $\theta=\pi \times$ (integer), by the chiral symmetry.

The next step is to dimensionally reduce this action to $(2+1) \mathrm{D}$ : We shrink the size of $z$-direction $L_{z}$ to zero, and decompose the vector field $V_{\mu}$ in $(3+1)$ d into vector and scalar fields in $(2+1) D$. Explicitly, we introduce the scalar in terms of the $z$ component of $V$ as $\Phi(u, x, y)=V_{z}(u, x, y) / L_{z}$. The resulting action is given by

$$
W_{\mathrm{eff}}[V]=\frac{\theta}{2 \pi^{2}} \int d u d x d y \varepsilon^{\mu \nu \lambda} \partial_{\mu} \Phi \partial_{\nu} V_{\lambda} .
$$

From the effective response action we can read off the topological responses and also topological invariants. (See, for example, Ref. [29]). We consider the magnetic field $B_{V}=\epsilon_{i j} \partial_{i} V_{j}$, and define the local magnetization density $M(u, x, y)$ by

$$
M(u, x, y) \equiv \frac{\delta W_{\mathrm{eff}}}{\delta B_{V}}=\frac{\theta}{2 \pi^{2}} \partial_{u} \Phi(u, x, y) .
$$

We also introduce the magnetization per unit volume

$$
m(u)=\frac{1}{V o l} \int d x d y M(u, x, y) .
$$

Then, the time average of $m(u)$ is

$$
\begin{aligned}
\overline{m(u)} & =\frac{1}{T} \int_{0}^{T} d u \frac{\theta}{2 \pi^{2}} \frac{d \Phi}{d u}=\frac{1}{T} \frac{\theta}{2 \pi^{2}}[\Phi(T)-\Phi(0)] \\
& =\frac{1}{T} \frac{\theta}{\pi} .
\end{aligned}
$$

Recalling $\theta=\pi \times$ integer, this fictitious magnetization is quantized. Its connection to the physical magnetization is unclear, though. Nevertheless, we note that it can be shown that the $\theta$ angle is given in terms of the winding number topological invariant associated with the unitary matrix $\mathcal{U}(t)$ [55]. This then proves, indirectly, that the fictitious magnetization agrees with the physical magnetization discussed in Ref. [22].

\section{APPENDIX C: PROOF OF QUANTIZATION OF $\theta$}

Here we give the details of the proof outlined in Sec. III E. In the below we will assume that the system is on a $L \times L$ torus rather than a cylinder, where the torus is obtained by gluing together the two boundaries of the cylinder of Fig. 4. This will not affect the value of $\theta$ since the lattice is populated only on a strip at a distance $d \gg \xi$ from the boundary of the cylinder.

\section{Magnetization operator}

Consider the projector $P^{\prime}$ on sites contained in the strip with $x$ in $(0, L-w)$, with $\xi \ll w \ll L$. Such region is then a subset of the strip with equal area, up to a correction of order $w / L$. Thanks to localization, this means that

$$
\operatorname{tr}\left(P_{\text {strip }} \mathcal{U}^{\dagger} \partial_{B} \mathcal{U}\right)=\operatorname{tr}\left(P^{\prime} \mathcal{U}^{\dagger} \partial_{B} \mathcal{U}\right)+O(\ell w)
$$

where the reminder can be neglected, as the first term is $O(\ell L)$.

In the presence of a background gauge field, the singleparticle Hamiltonian $\mathcal{H}$ in the position eigenstate basis $|\vec{r}\rangle$ becomes

$$
\mathcal{H}_{a b} \rightarrow \mathcal{H}_{a b} e^{i \int_{b}^{a} d \vec{r} \cdot \vec{A}}, \quad \mathcal{H}_{a b}=\left\langle\vec{r}_{a}|\mathcal{H}| \vec{r}_{b}\right\rangle .
$$

Taking the following gauge potential $\left(A_{x}, A_{y}\right)=\frac{B}{2}(-y, x)$, we have $\int_{b}^{a} d \vec{r} \cdot \vec{A}=\frac{B}{2}\left(y_{a} x_{b}-x_{a} y_{b}\right)$, which is well defined in the region covered by the projector $P^{\prime}$. Thus

$$
\begin{aligned}
\left.\partial_{B}\left(\mathcal{H}_{a b} e^{i \int_{a}^{b} d \vec{r} \cdot \vec{A}}\right)\right|_{B=0} & =i \mathcal{H}_{a b}\left(x_{b} y_{a}-x_{a} y_{a}\right) \\
& =-\frac{i}{2}\left\langle\vec{r}_{a}|\vec{r} \times[\vec{r}, H]| \vec{r}_{b}\right\rangle,
\end{aligned}
$$

where we used $\vec{r}\left|\vec{r}_{a}\right\rangle=\vec{r}_{a}\left|\vec{r}_{a}\right\rangle$, and $\vec{r}=(x, y)$. Then

$$
\begin{aligned}
\operatorname{tr}\left(P^{\prime} \mathcal{U}^{\dagger} \partial_{B} \mathcal{U}\right) & =-i \int_{-\kappa T / 2}^{\kappa T / 2} d t \operatorname{tr}\left(P^{\prime} \mathcal{U}^{\dagger}(t) \partial_{B} \mathcal{H}(t) \mathcal{U}(t)\right) \\
& =i \int_{-\kappa T / 2}^{\kappa T / 2} d t \operatorname{tr}\left[P^{\prime} \vec{r}(t) \times \partial_{t} \vec{r}(t)\right]
\end{aligned}
$$

where we used $\partial_{t} \mathcal{O}(t)=i\left[\mathcal{U}^{\dagger}(t) \mathcal{H}(t) \mathcal{U}(t), \mathcal{O}(t)\right]$ and where $\mathcal{U}(t)$ is the single-particle unitary from $-\kappa T / 2$ to $t$. Equation (C4) is the expectation value of the magnetization operator. 


\section{Coupling to $U$ (1) fluxes}

Consider again coupling the Hamiltonian to a background as in (C2), where this time we take a constant gauge field $\vec{A}=$ $\left(A_{x}, A_{y}\right)$. This gives $\int_{b}^{a} d \vec{r} \cdot \vec{A}=\vec{A} \cdot\left(\vec{r}_{a}-\vec{r}_{b}\right)$, and thus

$$
\frac{\partial\left(\mathcal{H}_{a b} e^{i \vec{A} \cdot\left(\vec{r}_{a}-\vec{r}_{b}\right)}\right)}{\partial \vec{A}}=i \vec{r}_{a} \mathcal{H}_{a b}-i \vec{r}_{b} \mathcal{H}_{a b},
$$

or

$$
\frac{\partial \mathcal{H}}{\partial \vec{A}}=i[\vec{r}, \mathcal{H}(t)]
$$

Defining

$$
\Delta \vec{r}(t)=-i \mathcal{U}^{\dagger} \partial_{\vec{A}} \mathcal{U}(t),
$$

the above implies that $\partial_{t} \Delta \vec{r}(t)=\partial_{t} \vec{r}(t)$. Since, from Eq. (C7), $\Delta \vec{r}(-\kappa T / 2)=0$,

$$
\Delta \vec{r}(t)=\vec{r}(t)-\vec{r}(-\kappa T / 2) .
$$

We have

$$
\begin{gathered}
\int_{-\kappa T / 2}^{\kappa T / 2} d t \operatorname{tr}\left(P^{\prime} \mathcal{U}^{\dagger}(t) \partial_{\vec{A}} \mathcal{U}(t) \times \mathcal{U}^{\dagger}(t) \partial_{\vec{A}} \mathcal{H U}\right) \\
=-i \int_{-\kappa T / 2}^{\kappa T / 2} d t \operatorname{tr}\left[P^{\prime} \Delta \vec{r}(t) \times \partial_{t} \vec{r}(t)\right] .
\end{gathered}
$$

Now

$$
\begin{aligned}
& -i \int_{-\kappa T / 2}^{\kappa T / 2} d t \operatorname{tr}\left[P^{\prime} \Delta \vec{r}(t) \times \partial_{t} \vec{r}(t)\right] \\
& =-i \int_{-\kappa T / 2}^{\kappa T / 2} d t \operatorname{tr}\left[P^{\prime} \vec{r}(t) \times \partial_{t} \vec{r}(t)\right] \\
& \quad+i \operatorname{tr}\left[P^{\prime} \vec{r}(-\kappa T / 2) \times \vec{r}(\kappa T / 2)\right] .
\end{aligned}
$$

The second term is bounded as a function of $\kappa$, as $\vec{r}$ can only take values within the strip. The definition of $\theta$ in (59) contains a factor of $\frac{1}{\kappa}$, and thus we can drop such term when taking $\kappa \rightarrow \infty$. We then find

$$
\begin{gathered}
\int_{-\kappa T / 2}^{\kappa T / 2} d t \operatorname{tr}\left[P^{\prime} \mathcal{U}^{\dagger}(t) \partial_{\vec{A}} \mathcal{U}(t) \times \mathcal{U}^{\dagger}(t) \partial_{\vec{A}} \mathcal{H} \mathcal{U}\right] \\
=-i \int_{-\kappa T / 2}^{\kappa T / 2} d t \operatorname{tr}\left[P^{\prime} \vec{r}(t) \times \partial_{t} \vec{r}(t)\right] .
\end{gathered}
$$

Using (C1), (C4), and (C2), we conclude

$$
\begin{aligned}
\operatorname{tr} & \left(P \mathcal{U}^{\dagger} \partial_{B} \mathcal{U}\right) \\
& =-\int_{-\kappa T / 2}^{\kappa T / 2} d t \operatorname{tr}\left[P^{\prime} \mathcal{U}^{\dagger}(t) \partial_{\vec{A}} \mathcal{U}(t) \times \mathcal{U}^{\dagger}(t) \partial_{\vec{A}} \mathcal{H} \mathcal{U}\right] \\
& =-\int_{-\kappa T / 2}^{\kappa T / 2} d t \operatorname{tr}\left[P_{\text {strip }} \mathcal{U}^{\dagger}(t) \partial_{\vec{A}} \mathcal{U}(t) \times \mathcal{U}^{\dagger}(t) \partial_{\vec{A}} \mathcal{H} \mathcal{U}\right] .
\end{aligned}
$$

Now consider a gauge field configuration

$$
\vec{A}=\left(A_{x}^{\prime}, A_{y}(y)\right), \quad A_{y}(y)=\left\{\begin{array}{ll}
A_{y}^{\prime} & y \in \text { strip } \\
0 & \text { otherwise }
\end{array},\right.
$$

where $A_{x}^{\prime}$ is constant, and $A_{y}$ is equal to $A_{y}^{\prime}$ within the strip and zero otherwise. Now note that the operators in Eq. (C12),

$$
\partial_{A_{y}^{\prime}} \mathcal{H}, \quad \mathcal{U}^{\dagger}(t) \partial_{A_{y}^{\prime}} \mathcal{U}(t),
$$

are nonvanishing only within the strip, up to exponential corrections in units of the localization length $\xi$. For the first operator, this is true because the Hamiltonian is local, and $A_{y}^{\prime}$ is varied only within the strip. For the second operator, this is also true because the system is localized. For such a gauge field configuration, we can then get rid of the strip projector $P_{\text {strip }}$ in the trace, and write

$$
\begin{gathered}
\operatorname{tr}\left[P_{\text {strip }} \mathcal{U}^{\dagger}(t) \partial_{\overrightarrow{A^{\prime}}} \mathcal{U}(t) \times \mathcal{U}^{\dagger}(t) \partial_{\vec{A}^{\prime}} \mathcal{H} \mathcal{U}\right] \\
=\operatorname{tr}\left[\partial_{\vec{A}^{\prime}} \mathcal{U}(t) \times \mathcal{U}^{\dagger}(t) \partial_{\vec{A}^{\prime}} \mathcal{H}\right],
\end{gathered}
$$

up to a term of order $L \xi$, where sites are populated with a fraction between 0 and 1 . Finally, the background (C13) is equivalent to threading fluxes $A_{x}^{\prime} L$ and $A_{y}^{\prime} \ell$ through the two holes of the torus. Similarly, the configuration used around (C5) is equivalent to fluxes $A_{x} L$ and $A_{y} L$. The two backgrounds are gauge equivalent if the respective fluxes are the same, i.e., $A_{x}^{\prime}=A_{x}$ and $A_{y}^{\prime}=A_{y} L / \ell$. This means that

$\operatorname{tr}\left[\partial_{\vec{A}} \mathcal{U}(t) \times \mathcal{U}^{\dagger}(t) \partial_{\vec{A}^{\prime}} \mathcal{H}\right]=\frac{\ell}{L} \operatorname{tr}\left[\partial_{\vec{A}} \mathcal{U}(t) \times \mathcal{U}^{\dagger}(t) \partial_{\vec{A}} \mathcal{H}\right]$.

Putting together (59), (C12), (C15), and (C16), we then find

$$
\theta=\frac{i \pi}{2 \kappa L^{2}} \int_{-\kappa T / 2}^{\kappa T / 2} d t \operatorname{tr}\left[\partial_{\vec{A}} \mathcal{U}(t) \times \mathcal{U}^{\dagger}(t) \partial_{\vec{A}} \mathcal{H}\right] .
$$

Note that the dependence on the strip size $\ell$ completely dropped out.

As a consequence of our effective response theory, in the thermodynamic limit the RHS of (C17) must be independent of $\vec{A}$ [56]. We can thus take its average

$$
\theta=\frac{i \pi}{2 \kappa L^{2}} \int_{-\kappa T / 2}^{-\kappa T / 2} d t\left(\frac{L}{2 \pi}\right)^{2} \int_{0}^{2 \pi / L} d^{2} A \operatorname{tr}\left[\partial_{\vec{A}} \mathcal{U}(t) \times \mathcal{U}^{\dagger}(t) \partial_{\vec{A}} \mathcal{H}\right]
$$

Integrating by parts we obtain

$$
\begin{aligned}
\theta= & \frac{i}{8 \pi \kappa} \int d t\left\{-\int d^{2} A \operatorname{tr}\left[\partial_{\vec{A}} \mathcal{U}(t) \times \partial_{\vec{A}} \mathcal{U}^{\dagger}(t) \mathcal{H}\right]\right. \\
& \left.+\left[\varepsilon^{i j} \int d A_{i} \operatorname{tr}\left(\partial_{A_{i}} \mathcal{U}(t) \mathcal{U}^{\dagger}(t) \mathcal{H}\right)\right]_{A_{j}=0}^{A_{j}=2 \pi / L}\right\} .
\end{aligned}
$$

Since $A_{j}=0$ and $A_{j}=2 \pi / L$ differ by a gauge transformation, we can write $\mathcal{H}\left(A_{j}=0, t\right)=X_{j}^{\dagger} \mathcal{H}\left(A_{j}=2 \pi / L, t\right) X_{j}$ and $\mathcal{U}\left(A_{j}=0, t\right)=X_{j}^{\dagger} \mathcal{U}\left(A_{j}=2 \pi / L, t\right) X_{j}$. Also, $\partial_{A_{i}} X_{j}=0$ for $i \neq j$, and thus the boundary term in (C19) vanishes. Using $\partial_{t} \mathcal{U}=-i \mathcal{H} \mathcal{U}$ and $\delta \mathcal{U} \mathcal{U}^{\dagger}=-\mathcal{U} \delta \mathcal{U}^{\dagger}$, with $\delta \mathcal{U}$ denoting a variation of $\mathcal{U}$, we then find

$$
\begin{aligned}
\theta= & -\frac{1}{8 \pi \kappa} \int_{-\kappa T / 2}^{\kappa T / 2} d t \int_{0}^{2 \pi / L} d^{2} A \\
& \times \operatorname{tr}\left[\mathcal{U}^{\dagger} \partial_{\bar{A}} \mathcal{U}(t) \times \mathcal{U}^{\dagger}(t) \partial_{\bar{A}} \mathcal{U}(t) \cdot \mathcal{U}^{\dagger}(t) \partial_{t} \mathcal{U}\right] .
\end{aligned}
$$

This looks like a winding number but $\mathcal{U}(t)$ is not periodic. In the next section we shall periodize the evolution. 


\section{Periodization and winding number}

Let us divide the time integral in (C20) into single-period intervals

$$
\theta=-\frac{\pi}{\kappa} \sum_{n=0}^{\kappa-1} \bar{W}\left[\mathcal{U}_{n}\right]
$$

where

$$
\begin{aligned}
\bar{W}\left[\mathcal{U}_{n}\right]= & \frac{1}{8 \pi^{2}} \int_{0}^{T} d t \int_{0}^{2 \pi / L} d^{2} A \\
& \times \operatorname{tr}\left[\mathcal{U}_{n}^{\dagger} \partial_{\vec{A}} \mathcal{U}_{n}(t) \times \mathcal{U}_{n}^{\dagger}(t) \partial_{\vec{A}} \mathcal{U}_{n}(t) \cdot \mathcal{U}_{n}^{\dagger}(t) \partial_{t} \mathcal{U}_{n}\right]
\end{aligned}
$$

where

$$
\mathcal{U}_{n}(t)=\mathcal{U}\left[t+\left(n-\frac{\kappa}{2}\right) T\right],
$$

and we further decompose $\mathcal{U}\left[t+\left(n-\frac{\kappa}{2}\right) T\right]=\mathcal{U}_{0}(t) \mathcal{U}((n-$ $\left.\left.\frac{\kappa}{2}\right) T\right)$, with $\mathcal{U}_{0}(t=0)=I$. Defining $\tilde{\mathcal{U}}_{n}(t)=\tilde{\mathcal{U}}_{0}(t) \mathcal{U}((n-$ $\left.\frac{\kappa}{2}\right) T$ ), with

$$
\tilde{\mathcal{U}}_{0}(t)=\left\{\begin{array}{ll}
\mathcal{U}_{0}(t) & t \in(0, T) \\
e^{i \mathcal{H}_{F}(t-2 T)} & t \in(T, 2 T)
\end{array},\right.
$$

where $\mathcal{H}_{F}$ is the Floquet Hamiltonian, defined through $e^{-i \mathcal{H}_{F} T}=\mathcal{U}_{0}(T)$, we see that $\tilde{\mathcal{U}}_{0}(t)$ is periodic with period $2 T$. Now write

$$
\bar{W}\left[\mathcal{U}_{n}\right]=W\left[\tilde{\mathcal{U}}_{n}\right]-W\left[e^{i \mathcal{H}_{F}(t-(n+1) T)}\right],
$$

where $W[\mathcal{U}]$ is defined as $\bar{W}[\mathcal{U}]$ in (C22) but with time integration over $(0,2 T)$. We now show that the second term in (C25) vanishes in the $t \rightarrow \infty$ limit. Decompose

$$
\mathcal{H}_{F}=\sum_{k} \varepsilon_{k} P_{k}, \quad e^{i \mathcal{H}_{F} t}=\sum_{k} e^{i \varepsilon_{k} t} P_{k},
$$

where $\varepsilon_{k}$ are the Floquet quasienergies and $P_{k}$ are projectors on the corresponding Floquet eigenstates, with $P_{k_{1}} P_{k_{2}}=0$ for $k_{1} \neq k_{2}$. Since Floquet eigenstates are localized, they will not wrap the cylinder, and the flux induced by $\vec{A}$ is thus felt as a gauge transformation. The quasienergies are therefore independent of $\vec{A}$, and the projectors $P_{k}$ depend on $\vec{A}$ as $\left(P_{k}\right)_{a b} e^{i \vec{A} \cdot\left(\vec{r}_{a}-\vec{r}_{b}\right)}$, where we are using the same notation as around Eq. (C5). This leads to

$$
\partial_{\vec{A}} P_{k}=i\left[\vec{r}, P_{k}\right]
$$

Then

$$
\begin{aligned}
& \sum_{n=0}^{\kappa-1} \bar{W}\left[e^{i \mathcal{H}_{F}(t-(n+1) T)}\right] \\
&=\frac{1}{8 \pi^{2}} \int_{0}^{2 \pi / L} d^{2} A \int_{-\kappa T}^{0} d t \\
& \quad \times \operatorname{tr}\left(e^{-i \mathcal{H}_{F} t} \partial_{\vec{A}} e^{i \mathcal{H}_{F} t} \times e^{-i \mathcal{H}_{F} t} \partial_{\vec{A}} e^{i \mathcal{H}_{F} t} \cdot i \mathcal{H}_{F}\right) \\
&=-\frac{i}{8 \pi^{2}} \epsilon^{i j} \int_{0}^{2 \pi / L} d^{2} A \int_{-\kappa T}^{0} d t \\
& \times \sum_{\left\{k_{i}\right\}} e^{-i \Delta \varepsilon t} \varepsilon_{k_{5}} \operatorname{tr}\left(P_{k_{1}}\left[r_{i}, P_{k_{2}}\right] P_{k_{3}}\left[r_{j}, P_{k_{4}}\right] P_{k_{5}}\right),
\end{aligned}
$$

where $\Delta \varepsilon=\varepsilon_{k_{1}}-\varepsilon_{k_{2}}+\varepsilon_{k_{3}}-\varepsilon_{k_{4}}$. At large $\kappa$, the integral will grow slower than $\kappa$, unless $\Delta \varepsilon=0$. We assume that the quasienergy spectrum is nondegenerate, which we expect to be generically true for a given disorder realization and flux value. Then only terms with $k_{1}=k_{2}$ and $k_{3}=k_{4}$ or $k_{1}=k_{4}$ and $k_{2}=k_{3}$ remain. After some manipulation, the remaining contribution is

$$
\begin{aligned}
& -\frac{i}{4 \pi^{2}} \varepsilon^{i j} \int_{0}^{2 \pi / L} d^{2} A \int_{-\kappa T}^{0} d t \sum_{k_{1}, k_{2}} \varepsilon_{k_{1}} \operatorname{tr}\left(P_{k_{1}} r_{i} P_{k_{2}} r_{j}\right) \\
& =-\frac{i \kappa T}{L^{2}} \varepsilon^{i j} \sum_{k_{1}, k_{2}} \varepsilon_{k_{1}} \operatorname{tr}\left(P_{k_{1}} r_{i} P_{k_{2}} r_{j}\right) \\
& =-\frac{i \kappa T}{L^{2}} \varepsilon^{i j} \sum_{k_{1}} \varepsilon_{k_{1}} \operatorname{tr}\left(P_{k_{1}} r_{i} r_{j}\right)=0,
\end{aligned}
$$

where we used $\sum_{k} P_{k}=I$. We are finally left with

$$
\theta=-\frac{\pi}{\kappa} \sum_{n=0}^{\kappa-1} W\left[\tilde{\mathcal{U}}_{n}\right]
$$

where $W\left[\tilde{\mathcal{U}}_{n}\right]$ was defined below $(\mathrm{C} 25)$ as the winding number of the unitary $\tilde{\mathcal{U}}_{n}$, the latter being periodic by construction. Assuming that the Floquet Hamiltonian $H_{F}$ is topologically trivial, in each term of the sum, we can smoothly deform to the identity the factor $\mathcal{U}\left(\left(n-\frac{\kappa}{2}\right) T\right)=e^{-i \mathcal{H}_{F}\left(n-\frac{\kappa}{2}\right) T}$ inside $\tilde{\mathcal{U}}_{n}(t)=\tilde{\mathcal{U}}_{0}(t) \mathcal{U}\left(\left(n-\frac{\kappa}{2}\right) T\right)$, and thus write

$$
\theta=-\pi W\left[\tilde{\mathcal{U}}_{0}\right] .
$$

We thus identified $\theta$ with the winding number of Titum et al. and quantization follows.
[1] A. Eckardt, Colloquium: Atomic quantum gases in periodically driven optical lattices, Rev. Mod. Phys. 89, 011004 (2017).

[2] T. Oka and S. Kitamura, Floquet engineering of quantum materials, Annu. Rev. Condens. Matter Phys. 10, 387 (2019).

[3] F. Harper, R. Roy, M. S. Rudner, and S. L. Sondhi, Topology and broken symmetry in Floquet systems, arXiv:1905.01317.

[4] T. Oka and H. Aoki, Photovoltaic Hall effect in graphene, Phys. Rev. B 79, 081406(R) (2009).
[5] T. Kitagawa, E. Berg, M. Rudner, and E. Demler, Topological characterization of periodically driven quantum systems, Phys. Rev. B 82, 235114 (2010).

[6] J.-I. Inoue and A. Tanaka, Photoinduced Transition between Conventional and Topological Insulators in Two-Dimensional Electronic Systems, Phys. Rev. Lett. 105, 017401 (2010).

[7] N. H. Lindner, G. Refael, and V. Galitski, Floquet topological insulator in semiconductor quantum wells, Nat. Phys. 7, 490 (2011). 
[8] T. Kitagawa, T. Oka, A. Brataas, L. Fu, and E. Demler, Transport properties of nonequilibrium systems under the application of light: Photoinduced quantum Hall insulators without Landau levels, Phys. Rev. B 84, 235108 (2011).

[9] L. Jiang, T. Kitagawa, J. Alicea, A. R. Akhmerov, D. Pekker, G. Refael, J. I. Cirac, E. Demler, M. D. Lukin, and P. Zoller, Majorana Fermions in Equilibrium and in Driven Cold-Atom Quantum Wires, Phys. Rev. Lett. 106, 220402 (2011).

[10] D. Carpentier, P. Delplace, M. Fruchart, and K. Gawedzki, Topological Index for Periodically Driven Time-Reversal Invariant 2D Systems, Phys. Rev. Lett. 114, 106806 (2015).

[11] R. Roy and F. Harper, Abelian Floquet symmetry-protected topological phases in one dimension, Phys. Rev. B 94, 125105 (2016).

[12] C. W. von Keyserlingk, V. Khemani, and S. L. Sondhi, Absolute stability and spatiotemporal long-range order in Floquet systems, Phys. Rev. B 94, 085112 (2016).

[13] C. W. von Keyserlingk and S. L. Sondhi, Phase structure of onedimensional interacting Floquet systems. I. Abelian symmetryprotected topological phases, Phys. Rev. B 93, 245145 (2016).

[14] C. W. von Keyserlingk and S. L. Sondhi, Phase structure of onedimensional interacting Floquet systems. II. Symmetry-broken phases, Phys. Rev. B 93, 245146 (2016).

[15] D. V. Else and C. Nayak, Classification of topological phases in periodically driven interacting systems, Phys. Rev. B 93, 201103(R) (2016).

[16] A. C. Potter, T. Morimoto, and A. Vishwanath, Classification of interacting topological Floquet phases in one dimension, Phys. Rev. X 6, 041001 (2016).

[17] Z. Gong, C. Sünderhauf, N. Schuch, and J. I. Cirac, Classification of Matrix-Product Unitaries with Symmetries, Phys. Rev. Lett. 124, 100402 (2020).

[18] A. C. Potter and T. Morimoto, Dynamically enriched topological orders in driven two-dimensional systems, Phys. Rev. B 95, 155126 (2017).

[19] R. Roy and F. Harper, Floquet topological phases with symmetry in all dimensions, Phys. Rev. B 95, 195128 (2017).

[20] M. S. Rudner, N. H. Lindner, E. Berg, and M. Levin, Anomalous Edge States and the Bulk-Edge Correspondence for Periodically Driven Two-Dimensional System, Phys. Rev. X 3, 031005 (2013).

[21] P. Titum, E. Berg, M. S. Rudner, G. Refael, and N. H. Lindner, Anomalous Floquet-Anderson Insulator as a Nonadiabatic Quantized Charge Pump, Phys. Rev. X 6, 021013 (2016).

[22] F. Nathan, M. S. Rudner, N. H. Lindner, E. Berg, and G. Refael, Quantized Magnetization Density in Periodically Driven Systems, Phys. Rev. Lett. 119, 186801 (2017).

[23] H. C. Po, L. Fidkowski, T. Morimoto, A. C. Potter, and A. Vishwanath, Chiral Floquet Phases of Many-Body Localized Bosons, Phys. Rev. X 6, 041070 (2016).

[24] L. Fidkowski, H. C. Po, A. C. Potter, and A. Vishwanath, Interacting invariants for Floquet phases of fermions in two dimensions, Phys. Rev. B 99, 085115 (2019).

[25] R. Roy and F. Harper, Periodic table for Floquet topological insulators, Phys. Rev. B 96, 155118 (2017).

[26] T. Morimoto, H. C. Po, and A. Vishwanath, Floquet topological phases protected by time glide symmetry, Phys. Rev. B 95, 195155 (2017).
[27] X.-L. Qi, T. L. Hughes, and S.-C. Zhang, Topological field theory of time-reversal invariant insulators, Phys. Rev. B 78, 195424 (2008).

[28] K. Shiozaki, H. Shapourian, and S. Ryu, Many-body topological invariants in fermionic symmetry-protected topological phases: Cases of point group symmetries, Phys. Rev. B 95, 205139 (2017).

[29] K. Shiozaki, H. Shapourian, K. Gomi, and S. Ryu, Many-body topological invariants for fermionic short-range entangled topological phases protected by antiunitary symmetries, Phys. Rev. B 98, 035151 (2018).

[30] A. Kamenev, Field Theory of Non-Equilibrium Systems (Cambridge University Press, Cambridge, UK, 2011).

[31] P. Glorioso and H. Liu, Lectures on non-equilibrium effective field theories and fluctuating hydrodynamics, arXiv:1805.09331.

[32] P. Glorioso and H. Liu, The second law of thermodynamics from symmetry and unitarity, arXiv:1612.07705.

[33] S. Roy and A. Lazarides, Nonequilibrium quantum order at infinite temperature: Spatiotemporal correlations and their generating functions, Phys. Rev. B 98, 064208 (2018).

[34] P. Ponte, Z. Papić, F. Huveneers, and D. A. Abanin, Many-Body Localization in Periodically Driven Systems, Phys. Rev. Lett. 114, 140401 (2015).

[35] A. Lazarides, A. Das, and R. Moessner, Fate of Many-Body Localization under Periodic Driving, Phys. Rev. Lett. 115, 030402 (2015).

[36] D. A. Abanin, W. D. Roeck, and F. Huveneers, Theory of manybody localization in periodically driven systems, Ann. Phys. 372, 1 (2016).

[37] D. A. Abanin, W. De Roeck, and F. Huveneers, Exponentially Slow Heating in Periodically Driven Many-Body Systems, Phys. Rev. Lett. 115, 256803 (2015).

[38] D. Abanin, W. De Roeck, W. W. Ho, and F. Huveneers, A rigorous theory of many-body prethermalization for periodically driven and closed quantum systems, Commun. Math. Phys. 354, 809 (2017).

[39] T. Mori, T. Kuwahara, and K. Saito, Rigorous Bound on Energy Absorption and Generic Relaxation in Periodically Driven Quantum Systems, Phys. Rev. Lett. 116, 120401 (2016).

[40] T. Kuwahara, T. Mori, and K. Saito, Floquet-Magnus theory and generic transient dynamics in periodically driven many-body quantum systems, Ann. Phys. 367, 96 (2016).

[41] D. V. Else, B. Bauer, and C. Nayak, Prethermal Phases of Matter Protected by Time-Translation Symmetry, Phys. Rev. X 7, 011026 (2017).

[42] D. J. Luitz, R. Moessner, S. Sondhi, and V. Khemani, Prethermalization Without Temperature, Phys. Rev. X 10, 021046 (2020).

[43] E. Fradkin and F. A. Schaposnik, Chern-Simons Gauge Theories, Confinement, and the Chiral Spin Liquid, Phys. Rev. Lett. 66, 276 (1991).

[44] F. Harper and R. Roy, Floquet Topological Order in Interacting Systems of Bosons and Fermions, Phys. Rev. Lett. 118, 115301 (2017).

[45] S. Elitzur, Y. Frishman, E. Rabinovici, and A. Schwimmer, Origins of global anomalies in quantum mechanics, Nucl. Phys. B 273, 93 (1986). 
[46] F. Nathan, D. A. Abanin, N. H. Lindner, E. Berg, and M. S. Rudner, Hierarchy of many-body invariants and quantized magnetization in anomalous Floquet insulators, arXiv: 1907.12228.

[47] D. Gross, V. Nesme, H. Vogts, and R. F. Werner, Index Theory of One Dimensional Quantum Walks and Cellular Automata, Commun. Math. Phys. 310, 419 (2012).

[48] T. Qin, Q. Niu, and J. Shi, Energy Magnetization and the Thermal Hall Effect, Phys. Rev. Lett. 107, 236601 (2011).

[49] K. Nomura, S. Ryu, A. Furusaki, and N. Nagaosa, CrossCorrelated Responses of Topological Superconductors and Superfluids, Phys. Rev. Lett. 108, 026802 (2012).

[50] A. Gromov and A. G. Abanov, Thermal Hall Effect and Geometry with Torsion, Phys. Rev. Lett. 114, 016802 (2015).
[51] F. Tonielli, J. C. Budich, A. Altland, and S. Diehl, Topological Field Theory Far from Equilibrium, Phys. Rev. Lett. 124, 240404 (2020).

[52] R. Dijkgraaf and E. Witten, Topological gauge theories and group cohomology, Commun. Math. Phys. 129, 393 (1990).

[53] X. Chen, Z.-C. Gu, Z.-X. Liu, and X.-G. Wen, Symmetry protected topological orders and the group cohomology of their symmetry group, Phys. Rev. B 87, 155114 (2013).

[54] H. Umezawa, Advanced Field Theory: Micro, Macro, and Thermal Physics (AIP College Park, MD, USA, 1995).

[55] S. Ryu, A. P. Schnyder, A. Furusaki, and A. W. W. Ludwig, Topological insulators and superconductors: Tenfold way and dimensional hierarchy, New J. Phys. 12, 065010 (2010).

[56] M. B. Hastings and S. Michalakis, Quantization of Hall conductance for interacting electrons on a torus, Commun. Math. Phys. 334, 433 (2015). 